\title{
Megacircones ediacáricos de la sienita nefelínica de San José del Guaviare y su potencial como material de referencia para datación U/Pb mediante LA-ICP-MS
}

\author{
Ediacaran mega-zircons of the San José del Guaviare nepheline syenite \\ and their potential use as reference material for U/Pb dating through \\ LA-ICP-MS
}

Jimmy Alejandro Muñoz Rocha ${ }^{1}$, Alejandro Piraquive ${ }^{1-3}$, José A. Franco ${ }^{2-3}$, Amed Bonilla ${ }^{2-3}$, Mary Luz Peña Urueña ${ }^{1}$, Thomas Cramer ${ }^{2-3}$, Lorena Rayo Rocha ${ }^{1}$, Nicolás Villamizar ${ }^{1}$

Recibido: 24 de julio de 2018

Aceptado: 18 de marzo de 2019

Publicado en línea: 15 de julio de 2019

Doi: 10.32685/0120-1425/boletingeo.45.2019.484

Citación: Muñoz Rocha, J. A., et al. (2019). Megacircones ediacáricos de la sienita nefelínica de San José del Guaviare y su potencial como material de referencia para datación U/Pb mediante LA-ICP-MS. Boletín Geológico, 45, 5-22. Doi: https://doi.org/10.32685/0120-1425/boletingeo.45.2019.484

\section{RESUMEN}

Con el objetivo de ampliar el conjunto de materiales de referencia del Laboratorio de Geocronología del Servicio Geológico Colombiano (LG-SGC) se colectaron circones provenientes de la sienita nefelínica de San José del Guaviare, localidad del Jordán (Colombia). La muestra SNG-1 se compuso de 24 circones seleccionados de la fracción situada entre 2 y 5 milímetros de largo, montados y pulidos en un bloque de resina epóxica. La datación se realizó mediante análisis de relaciones isotópicas de uranio y plomo, utilizando el método LA-ICPMS. En el LG-SGC se seleccionaron 92 puntos sobre los circones y en la Universidad de Rennes, en Francia (LG-URF), se analizaron 24 puntos en áreas cercanas de los mismos cristales mediante estudio previo de catodoluminiscencia. Las edades concordantes obtenidas de los circones corresponden, en ambos laboratorios, a la edad de cristalización de $608 \pm 1,2$ Ma y $602 \pm 3$ Ma, respectivamente, que difieren en menos del 1\%, pese a la diferencia en instrumentación y las rutinas analíticas. Para corrección de errores sistemáticos y control de calidad se emplearon materiales de referencia internacionales, como circones de Plešovice, GJ-1, FC-5 Duluth y Mount Dromedary. Las incertidumbres producidas durante los

\footnotetext{
Servicio Geológico Colombiano (SGC)

Universidad Nacional de Colombia

3 Grupo de Estudios en Geología Económica y Mineralogía Aplicada (Gegema)

Email de correspondencia: jamunoz@sgc.gov.co
} 
análisis son similares (1,2 y 3,2 Ma), y el parámetro MSWD de los diagramas de concordia fue $<1$ en ambos casos, lo que indica baja dispersión de los resultados. Algunas edades discordantes reveladas durante el análisis muestran que las relaciones $\mathrm{U} / \mathrm{Pb}$ no son homogéneas, como se espera en un material de referencia primario. Sin embargo, consideramos, que los circones de la sienita del Jordán tienen potencial como material de referencia secundario para el LG-SGC, siempre y cuando se emplee una cuidadosa selección y preparación de estos, se caracterice su contenido geoquímico, incluyendo los elementos de tierras raras (REE) y se ejecute un monitoreo de calidad de dataciones $\mathrm{U} / \mathrm{Pb}$, teniendo como base significativa el gran tamaño de los circones, que puede alcanzar los 4 $\mathrm{cm}$, su abundancia y posibilidad de recolección en el territorio colombiano.

Palabras clave: datación U/Pb de circones, láser, espectrometría de masas, megacircones, material referencia laboratorio, LA-ICPMS, geocronología, ediacárico, Colombia.

\begin{abstract}
With the aim of expanding the reference materials of the Geochronology Laboratory of the Colombian Geological Survey (LG-SGC, in Spanish), zircons were collected from the nepheline syenite of San José del Guaviare, Jordán locality (Colombia). The SNG-1 sample was composed of 24 zircons selected from the fraction between 2 and 5 millimeters long, which were mounted and polished in an epoxy resin block. The zircons were dated by analyzing the isotopic ratios of uranium and lead using the LA-ICPMS method. In the LG-SGC, 92 points on the zircons were selected for analysis, and at the University of Rennes in France (LG-URF), 24 points from crystals found nearby were selected for analysis from a previous cathodoluminescence study. Concordant ages for the zircons for both laboratories corresponded to a crystallization age of $608 \pm 1.2 \mathrm{Ma}$ and $602 \pm 3 \mathrm{Ma}$, respectively, which differ by less than $1 \%$ despite the difference in instrumentation and analytical procedures. For systematic error correction and quality control, international reference material, such as Plešovice, GJ-1, FC-5 Duluth and Mount Dromedary zircon, was used. The uncertainties generated during the analyses are similar (1.2 and 3.2 Ma), and the mean square-weighted deviation (MSWD) of the concordia diagrams was $<1$ in both cases, indicating low dispersion of the results. Some discordant ages show that the $\mathrm{U} / \mathrm{Pb}$ ratios are not uniform, as expected for a primary reference material. However, we believe that the zircons of the Jordán syenite have potential use as a secondary reference material for the LG-SGC provided they are carefully selected and prepared, their geochemical content (including rare earth elements, REE) is characterized, and quality monitoring of $\mathrm{U} / \mathrm{Pb}$ dating is performed, as these zircons are large, reaching up to $4 \mathrm{~cm}$, abundant, and possible to collect in the Colombian territory.

Keywords: U/Pb dating of zircons, laser, mass spectrometry, mega-zircons, laboratory reference material, LAICPMS, geochronology, Ediacaran, Colombia.
\end{abstract}

\section{INTRODUCCIÓN}

ara la datación de materiales geológicos, el sistema isotópico U-Th- $\mathrm{Pb}$ es uno de los más confiables y probados, permite determinar edades absolutas utilizando minerales con alto contenido de $\mathrm{U}$, como es el caso del circón (Corfu et al., 2003). El circón es un nesosilicato de circonio $\left[\mathrm{ZrSiO}_{4}\right]$ que se presenta como un mineral accesorio en rocas sedimentarias, metamórficas y sobre todo, rocas ígneas, donde se forma principalmente en plutonitas (granitoides). Debido a su alto punto de fusión (a partir de $1676{ }^{\circ} \mathrm{C}$ bajo presión normal se descompone incongruentemente en $\mathrm{ZrO}_{2}$ y $\mathrm{SiO}_{2}$ ) y gracias a su estabilidad estructural, con dureza de 7,5 Mohs (Finch y Hanchar, 2005), los circones pueden resistir variaciones de temperatura o de presión alta, al tiempo que incorporan 
y preservan las interacciones con cada nuevo ambiente geológico, que se reflejan en la forma, textura y composición química del mineral, lo que permite, por ejemplo, el uso de circones detríticos para rastrear el origen de rocas sedimentarias (Fedo, Sircombe y Rainbird, 2003).

La estructura cristalina tetragonal del circón con clase cristalina $4 / \mathrm{m} 2 / \mathrm{m} 2 / \mathrm{m}$ puede incorporar y retener elementos de tierras raras (REE), incluyendo Y, Sc y lantánidos, y sobre todo cationes de tamaño similar con valencia $4+$, como el Hf (normalmente entre 1 y $2 \%$, aunque puede llegar a 32\%), así como trazas de otros elementos (P, $\mathrm{Ti}, \mathrm{Th}, \mathrm{U})$. La isotopía de circón $\left(\mathrm{Zr}_{[}\left[\mathrm{SiO}_{4}\right]\right)$ y xenotima-Y $\left(\mathrm{Y}\left[\mathrm{PO}_{4}\right]\right)$ causa una sustitución acoplada (heterovalente) $\mathrm{Zr}^{4+}$ y $\mathrm{Si}^{4+}$ por $\mathrm{Y}^{3+}$ y $^{5+}$. Debido a la contracción de los lantánidos, $\mathrm{Zr}^{4+} \mathrm{y} \mathrm{Hf}^{4+}$ tienen radios iónicos casi idénticos, y se forma una solución sólida completa entre el hafnón $\left(\mathrm{Hf}\left[\mathrm{SiO}_{4}\right]\right)$ y el circón, lo que transforma los circones en la mena principal de hafnio. (Turekian y Wedepohl, 1961).

La tabla 1 muestra el enriquecimiento de $\mathrm{Zr}$ y Hf en rocas ácidas y sienitas junto con la relación $\mathrm{Zr} / \mathrm{Hf}$. Se observa cierta disminución de la relación $\mathrm{Zr} / \mathrm{Hf}$ en circones asociados a la diferenciación magmática, lo que explica inicialmente la abundancia e importancia de los circones asociados a la Sienita Nefelinica de San José del Guaviare (SNSJG), localidad del Jordan.

$\mathrm{El} \mathrm{U} \mathrm{U}^{4+} \mathrm{y} \mathrm{Th}^{4+}$ en coordinación 6 (tienen radios iónicos de 0,89 y $0,94 \AA$, respectivamente (Shannon, 1976), lo que, junto con sus altas valencias, los hace incompatibles para entrar en las estructuras cristalinas de los principales silicatos formadores de roca; en vez de eso, el $\mathrm{U}^{4+}$ con valores Clarke de 1,7 ppm en corteza continental (Wedepohl, 1995) forma minerales como la uranita o substituye al $\mathrm{Zr}^{4+}$ con radio iónico de $0,86 \AA$ y coordinación 6 en el circón $\left(\mathrm{ZrSiO}_{4}\right)$, al $\mathrm{Ti}^{4+}$ en el rutilo, al $\mathrm{Nb}$ y Ta en columbitas-tantalitas y por algunos cationes en minerales de REE, mientras que el Th con valores Clarke de 8,5 ppm en corteza continental (Wedepohl, 1995) forma minerales accesorios, como torianita $\left(\mathrm{ThO}_{2}\right)$ y torita $\left(\mathrm{ThSiO}_{4}\right)$, o se concentra principalmente en fosfatos como la monacita, y sustituye el $\mathrm{Zr}^{4+}$ en circones en menor medida. En coordinación 8, todos estos radios aumentan entre 10 y $14 \%$, y las reglas de Goldschmidt dejan constancia de que una variación de radios iónicos de hasta $15 \%$ permite un intercambio de cationes en la misma estructura cristalina sin problemas, si tienen la misma valencia, mientras diferencias de valencias $>1$, prácticamente inhiben la sustitución catiónica.

La incorporación de U y Th (de 10 ppm hasta 5\%) en circones, su alta resistencia, su presencia en casi todos los ambientes geológicos y la escaza incorporación de plomo común, los hicieron durante décadas el mineral preferido para dataciones $\mathrm{U} / \mathrm{Pb}$, aunque en los últimos años, gracias al desarrollo, sobre todo de LA-ICP-MS, también muchos otros minerales que incorporan trazas de $\mathrm{U}$ y Th han mostrado su utilidad geocronológica. En el caso de los circones, la metamictización (isotropización) causada por el decaimiento radiactivo de $\mathrm{U}$ y Th puede bajar su densidad normal de 4,6 a $4,7 \mathrm{~g} / \mathrm{cm}^{3}$ a valores entre 3,9 y $4,2 \mathrm{~g} / \mathrm{cm}^{3}$ ("low density zircons"). La medición de la relación de isótopos de uranio y torio y de isótopos de plomo permite su uso como un reloj natural, y la datación de eventos geológicos ocurridos desde la formación de la Tierra, hace 4,57 Ga, hasta el Pleistoceno (Schoene, 2014).

Los circones muestran a menudo exsoluciones e inclusiones y, algo especialmente importante, una zonación química y textural correspondiente a diferentes etapas de cristalización durante procesos magmáticos, hidrotermales y metamórficos (e. G., núcleos heredados, anillos), la que se puede observar bajo el microscopio petrográfico (mejorado con cauterización HF) y sobre todo con catodoluminiscencia pancromática, que es sensible a la presencia de ciertos elementos traza y defectos en la estructura cristalina (Koschek, 1993; Yang, Luff y Townsend 1992;

Tabla 1. Enriquecimiento de $\mathrm{Zr}$ y Hf en rocas ácidas, sienitas y relación $\mathrm{Zr} / \mathrm{H}$

\begin{tabular}{cccc}
\hline & Zr [ppm] en rocas & Zr/Hf en circones & Hf [ppm] en rocas \\
\hline Rocas ultrabásicas & 45 & 70 & \\
\hline Gabro & 140 & 70 & \\
\hline Granodiorita & 140 & 60 & \\
\hline Granito & 175 & 45 & \\
\hline Sienita & 500 & 45 & 3,0 \\
\hline Rocas sedimentarias & & Zr/Hf $=55$ & 11,1 \\
\hline
\end{tabular}

Fuente: Turekian y Wedepohl (1961) 
Hanchar y Miller, 1993). El contenido de Ti en circones cristalizados en un ambiente saturado en Ti depende en gran medida de la temperatura y se utiliza como un geotermómetro (Watson y Harrison, 2005; Watson, Wark y Thomas, 2006; Harrison, Watson y Aikman, 2007). Hay que tener en cuenta todos estos factores para la correcta aplicación e interpretación de dataciones radiométricas en circones, entre otras razones porque pueden modificar la temperatura de cierre de unos $900{ }^{\circ} \mathrm{C}$, por debajo de la cual se supone que en los circones no habrá pérdidas de isótopos de $\mathrm{U}$, Th y $\mathrm{Pb}$, como base fundamental para poder realizar dataciones confiables analizando series de decaimiento, como ${ }^{238} \mathrm{U} \rightarrow{ }^{206} \mathrm{~Pb},{ }^{235} \mathrm{U} \rightarrow{ }^{207} \mathrm{~Pb}$ o ${ }^{232} \mathrm{Th} \rightarrow{ }^{208} \mathrm{~Pb}$, según la ecuación de decaimiento $\mathrm{N}=$ $\mathrm{n}_{0} \mathrm{e}^{-\lambda t}$, donde $\mathrm{N}$ es el número de isótopos al tiempo t, $\mathrm{N}_{0}$ es el momento del cierre del sistema, y $\lambda$ la constante de decaimiento característica de cada isótopo, un principio reconocido y aplicado desde los trabajos de Rutherford y Soddy (1903). En el sistema U/Th/Pb utilizamos ecuaciones derivadas como la siguiente:

$$
\frac{\Delta\left({ }^{207} \mathrm{~Pb} /{ }^{204} \mathrm{~Pb}\right)}{\Delta\left({ }_{206} P b /{ }^{204} \mathrm{~Pb}\right)}=\frac{\left(e^{\lambda 235 t}-1\right)}{137,82\left(e^{1238 t}-1\right)}
$$

Siendo 137,82 la relación ${ }^{1}{ }^{238} \mathrm{U} /{ }^{235} \mathrm{U}$, que resolvemos iterativamente con algoritmos de minimización (regresión). La relación de plomo radiógeno ${ }^{207} \mathrm{~Pb} /{ }^{206} \mathrm{~Pb}$ tiene la ventaja adicional de no verse afectada por difusión de $\mathrm{U}$ ni de $\mathrm{Pb}$ del sistema.

El uso de diagramas de concordia en vez de isócronas (véase el capítulo "Resultados") permite adicionalmente eliminar ${ }^{204} \mathrm{~Pb}$ del cálculo de las edades.

Entre las diferentes técnicas geocronológicas que existen para estudiar y datar circones, la técnica LA-ICP-MS está en un proceso de continuo desarrollo desde 1985 (p. Ej., Košler y Sylvester, 2003), entre otras razones porque con esta técnica se realizan análisis in situ de alta resolución local con ablaciones de algunas decenas de micrómetros sobre la superficie de circones pulidos, lo cual brinda resultados rápidos y precisos (Campbell, Reiners, Allen,
Nicolescu y Upadhyay, 2005; Solari, Gómez, Bernal, Pérez y Tanner, 2010). Además, una vez instalada y probada, es una técnica de bajo costo, rápida (aproximadamente 2 minutos por análisis) y bastante precisa, con un error interno de $\sim 1 \%$ o menor (para dataciones $\mathrm{U}-\mathrm{Pb}$ ), por lo que tiene una amplia aplicación en las geociencias, lo cual se ha traducido en un crecimiento exponencial, en las últimas décadas, del número de publicaciones de edades $\mathrm{U}-\mathrm{Pb}$ en circones (Schoene, 2014).

Con el fin de asegurar la calidad analítica de la técnica LA-ICP-MS se usan materiales de referencia para detectar y corregir errores sistemáticos, como el fraccionamiento elemental, y para verificar la exactitud y precisión de los resultados de medición. Los resultados de la medición adecuada de los materiales de referencia indican si la técnica fue aplicada correctamente para determinar edades en la muestra desconocida (Chang, Vervoort, mcclelland y Knaack, 2006).

No obstante, existe un número limitado de materiales de referencia utilizados como estándares primarios e internos que cumplen con las condiciones necesarias para ser considerados como tales, es decir, que sean homogéneos en cuanto a su contenido de U, no presentan daño metamíctico o hidrotermal, y sus edades presentan una población unimodal con una baja dispersión.

Debido a que el Laboratorio de Geocronologia del Servicio Geológico Colombiano (LG-SGC) es relativamente nuevo, es de suma importancia en la implementación confiable de la técnica adquirir y utilizar diferentes materiales de referencia durante la realización de dataciones de circón. La mayoría de materiales estándares utilizados en este laboratorio provienen de regiones de Europa, Norteamérica y Asia, y no se cuenta en este momento con un material de referencia, primario o secundario, que provenga de Suramérica. Esto significa que para suplir las necesidades del Laboratorio de Datación U-Pb del SGC, que debe estar en un continuo funcionamiento para datar muestras del territorio colombiano, es necesario importar frecuentemente estos materiales de referencia.

En esta contribución presentamos los resultados del análisis de circones extraídos de la sienita nefelínica

1. En los últimos años se encontró que esta relación puede variar un poco, y es también por debajo del valor "oficial" de 137,88 recomendado por la IGC Subcommission on Geochronology, lo que, igualmente como variaciones de l, puede causar desviaciones sistemáticas en el cálculo de las edades isotópicas. 
de San José del Guaviare, en la localidad del Jordán. Por su abundancia, inusual gran tamaño y contenido de elementos de tierras raras (REE), presentan una oportunidad única de estudio para entender su formación y establecer si pueden ser utilizados como material de referencia del laboratorio. La facilidad de su adquisición, por ser un material propio de nuestro territorio, resultaría en una gran oportunidad para asegurar la continua producción de información geocientífica en el Laboratorio de Geocronologia del Servicio Geológico.

Además, se llevaron a cabo análisis comparativos en el LG-SGC y en el Laboratorio de Géochimie Isotopique et Géochronologie de l'Université de Rennes (Francia). Este último es un referente a nivel mundial en geocronología de U-Th- $\mathrm{Pb}$, donde se datan, con LA-ICP-MS, diferentes minerales, como circón, monacita, esfena, badeleyita, xenotima, rutilo, alanita, apatito, calcita, columbita-tan- talita y scheelita. Por medio de este ejercicio se buscó verificar si las edades de estos cristales obtenidas en ambos laboratorios son similares, y si los resultados son precisos y exactos, algo que discutiremos a continuación.

\section{Descripción de la muestra de CIRCONES}

Los circones fueron extraídos de la sienita nefelínica recolectada en la localidad del Jordán, en el departamento del Guaviare, en el centro-oriente de Colombia (figura 1). Este cuerpo plutónico presenta variaciones locales a pegmatitas y aplitas y está constituido principalmente por feldespato (microclina), nefelina, biotita y hornblenda (figura 2) (Arango, Nivia, Zapata, Giraldo, Bermúdez y Albarracín, 2011; Arango, Zapata y Martens, 2012; Trumpy, 1943; Campos Rodríguez, 2017; García Romero, 2015).

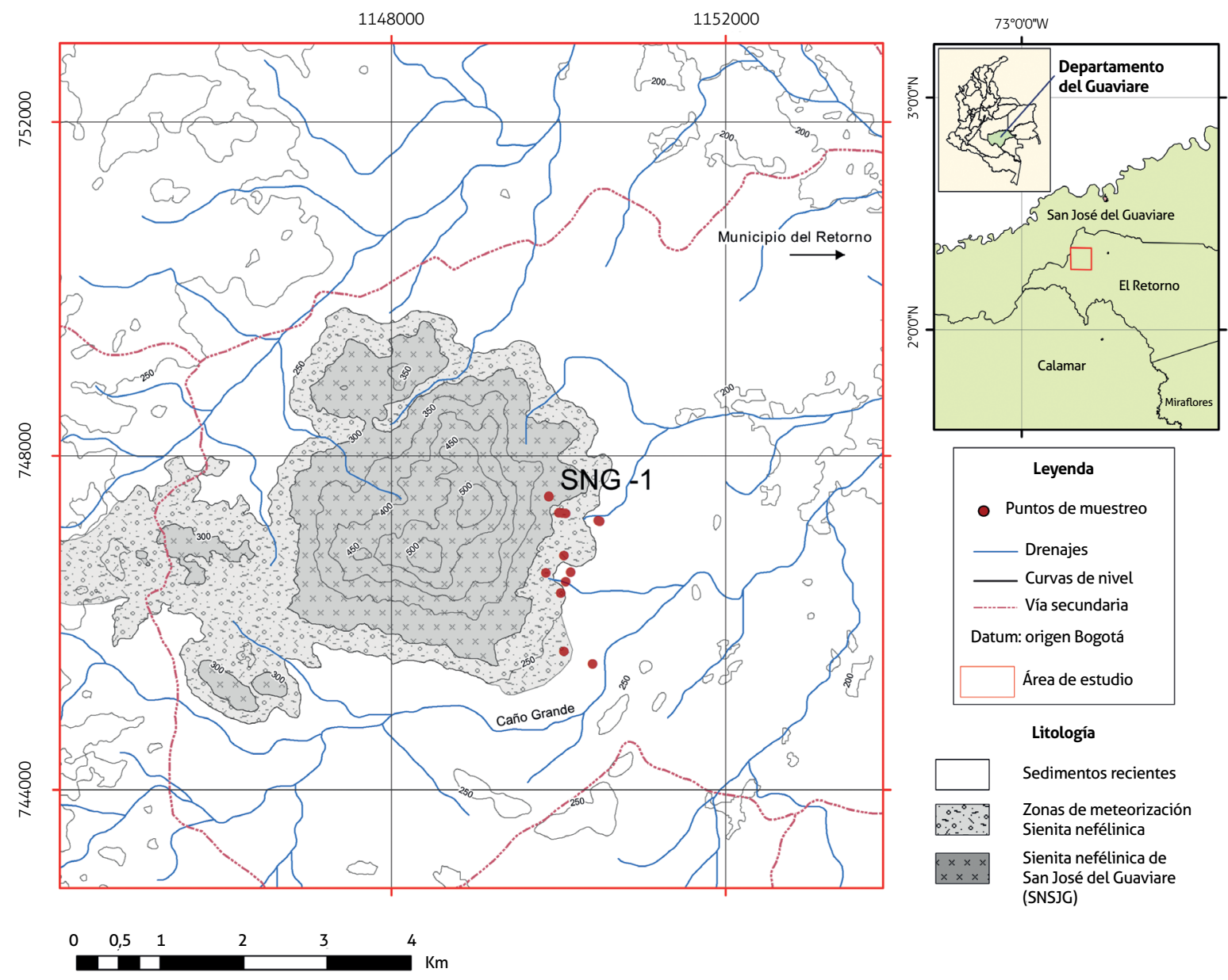

Figura 1. Mapa de localización de la sienita nefelínica de San José del Guaviare, localidad del Jordán, y de los puntos de toma de muestras de circones.

Fuente: autores 

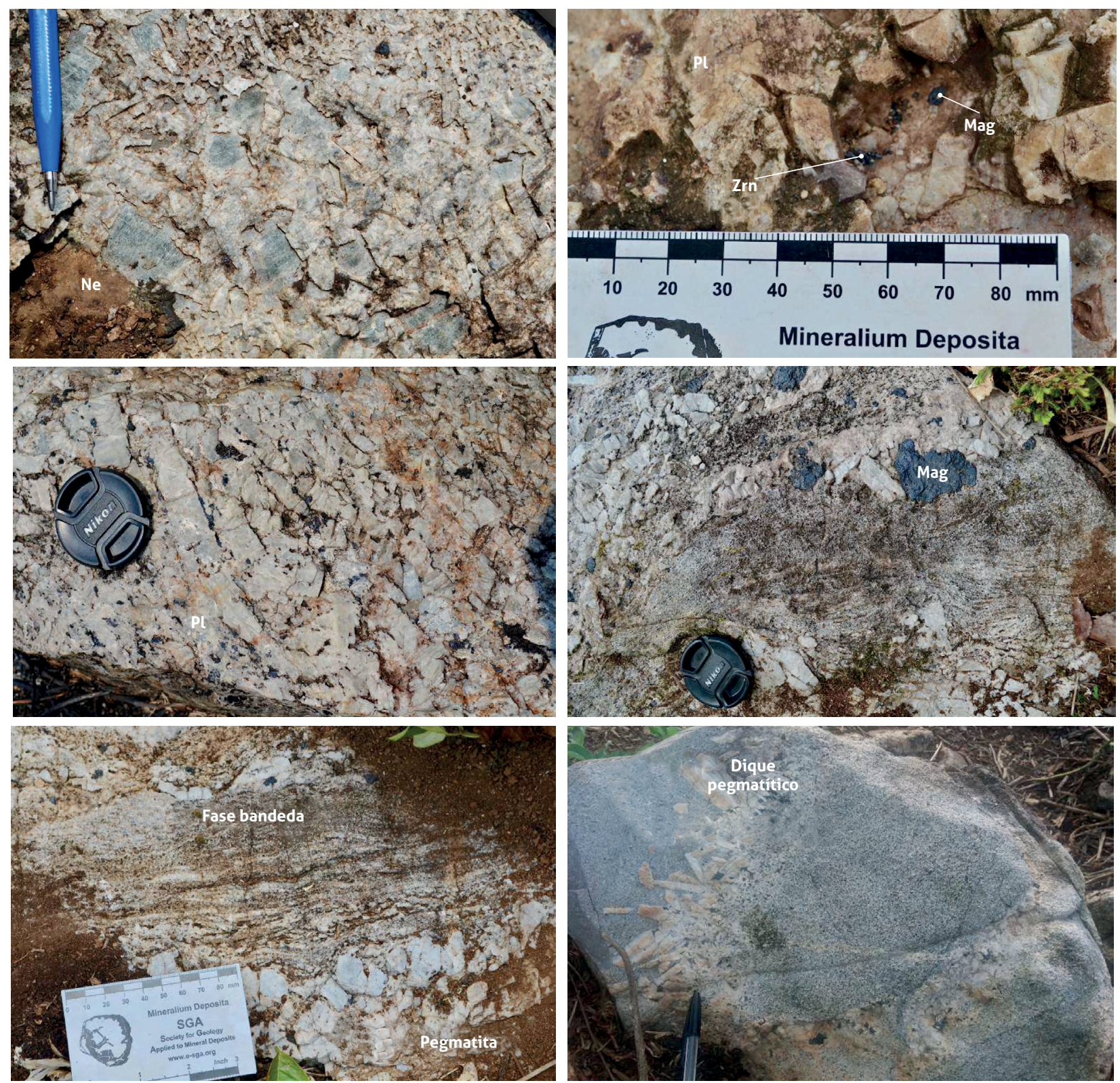

Figura 2. Compilación de características mesoscópicas de la sienita nefelínica. Fuente: autores

La figura 2 muestra las especies minerales en paragénesis. Son frecuentes las texturas pegmatíticas con megacristales de feldespatos, circón, nefelina, biotita, magnetita y anfíbol. La textura pegmatítica puede encontrarse en diques hacia las zonas de borde de domos graníticos. Esta fase más gruesa contiene los mayores cristales de circón y magnetita, que alcanzan hasta $4 \mathrm{~cm}$. La nefelina muestra una coloración grisácea y azulosa con un lustre perlado típico de alteración a cancrinita. Algunos secto- res de la sienita muestran minerales foliados y bandas de cizalla conjugadas que contienen biotita. Las relaciones de corte no son aun suficientes para definir si la lineación mineral es magmática, una lineación relíctica de la roca caja, o episodios de tectonismo durante y después del emplazamiento de sucesivas fases magmáticas; no obstante, los diques pegmatíticos son tardíos respecto al cuerpo principal (Franco, Muñoz, Piraquive, Bonilla, Cramer y Campos, 2018). 

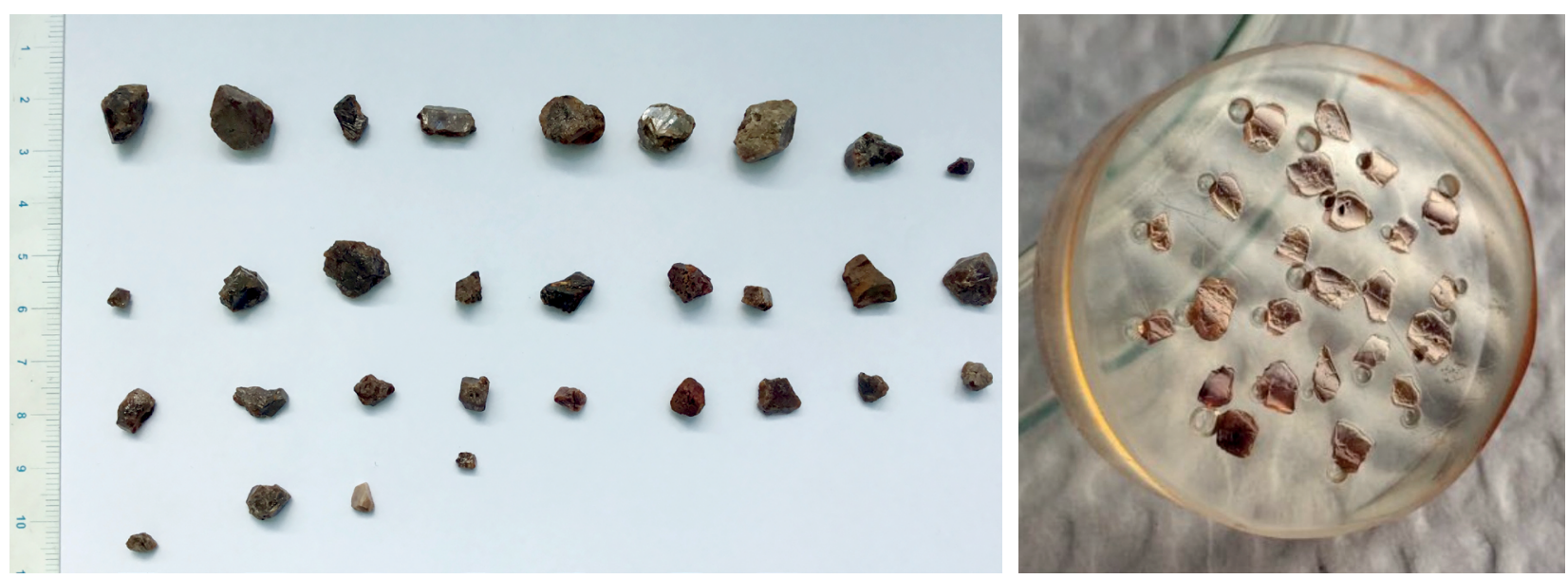

Figura 3. Izquierda: circones de la sienita nefelínica de San José del Guaviare, localidad del Jordán. Derecha: montaje estudiado, de $2,5 \mathrm{~cm}$ de diámetro.

Fuente: autores

Para el análisis geocronológico se seleccionaron circones en su mayoría prismáticos, con una longitud de 2 a 5 mm y una relación de longitud a ancho de 3:2 a 2:1 (figura 3), con algunos cristales hasta de 4:1. Se seleccionó esta fracción debido a que los cristales de tamaño superior a 2 $\mathrm{cm}$ exhiben daños metamícticos considerables causados por alto contenido de $\mathrm{U}$, lo que puede resultar en edades discordantes, mientras la fracción de menor tamaño es, en términos generales, más homogénea, y por eso, más adecuada para este estudio.

\section{Datación U-Pb mediante LA-ICP-MS}

La datación U-Pb de los circones mediante LA-ICP-MS se llevó a cabo en el Laboratorio de Geocronologia del Servicio Geológico Colombiano y en el Laboratorio de LA-ICPMS de la Universidad de Rennes. Solo aquellos circones de los que previamente se calcularon edades concordantes en el SGC fueron datados en el laboratorio de Rennes, y en puntos cercanos a los ablacionados en el LG-SGC.

Los detalles de adquisición de imágenes de catodoluminiscencia, ablación láser, parámetros del ICP-MS y reducción de datos empleados se muestran en la tabla 2. La reproducibilidad de las relaciones isotópicas $\mathrm{U}-\mathrm{Pb}$ fue calibrada, en el caso del Servicio Geológico Colombiano, utilizando el estándar primario Plesovice (edad 337,13 \pm
0,37 Ma, Sláma et al., 2008), mientras que los estándares FC-5 Duluth (edad 1.099 \pm 0,6 Ma, Paces y Miller, 1993) y Mount Dromedary (edad 99,12 \pm 0,14 Ma, Renne, Swisher, Deino, Karner, Owens y De Paolo, 1998) fueron utilizados como estándares secundario y terciario, respectivamente, para calibrar y monitorear la precisión y exactitud de las edades $\mathrm{U}-\mathrm{Pb}$ medidas.

En la Universidad de Rennes se utilizó el estándar de referencia primario GJ-1 con una edad de 607,7 \pm 0,67 Ma (Jackson, Pearson, Griffin y Belousova, 2004), y para control de calidad, el estándar Plesovice. Los resultados de datación de circones mediante LA-ICP-MS se muestran en la tabla 2.

Antes de realizar los análisis mediante LA-ICP-MS se tomaron imágenes de catodoluminiscencia (figura 4). Estas imágenes muestran que la mayoría de los cristales idiomórficos presentan una zonación oscilatoria típica de origen ígneo (figura $4 \mathrm{a}-1$ ) y una alta concentración de $\mathrm{U}$, elementos trazas y tierras raras; la ausencia de núcleos heredados indica una cristalización durante un evento magmático continuo. Sin embargo, núcleos con zonación oscilatoria y sectorial en algunos cristales y, en algunas ocasiones con bordes de sobrecrecimiento discretos y zonas con moteado, indican mayor alteración termal y daños metamícticos (figura $4 \mathrm{~m}-\mathrm{p}$ ), lo que disminuye la relevancia de los datos obtenidos. 
Tabla 2. Instrumentación y parámetros de análisis utilizados

\begin{tabular}{|c|c|c|}
\hline \multicolumn{3}{|c|}{ Laboratorio y preparación de muestras } \\
\hline Nombre del laboratorio & $\begin{array}{l}\text { Laboratorio de Geocronologia, Servicio Geológico } \\
\text { Colombiano }\end{array}$ & \\
\hline Tipo de muestra o mineral & Zircones, sienita nefelínica & \\
\hline Preparación de la muestra & $\begin{array}{l}\text { Separación convencional de minerales (trituración, } \\
\text { batea, separación magnética, separación por líquidos } \\
\text { densos), selección de cristales manual, montaje de } \\
\text { resina epóxica de una pulgada y pulido con pasta de } \\
\text { diamante de } 6 \mu \mathrm{m}\end{array}$ & \\
\hline Imágenes & $\begin{array}{l}\text { Detector GATAN Minicl pancromático, acoplado a un } \\
\text { microscopio electrónico de barrido JEOL JSM-IT300LV, } \\
15 \text { kv, modo alta corriente }\end{array}$ & \\
\hline \multicolumn{3}{|c|}{ Sistema de ablación láser } \\
\hline & LG - SGC & Laboratorio Rennes \\
\hline Marca, modelo y tipo & Photon Machines, Exite & ESI, Excimer (NWR193C) \\
\hline Celda de ablación & Helex 2 & Helex 2 \\
\hline Longitud de onda láser & $193 \mathrm{~nm}$ & $193 \mathrm{~nm}$ \\
\hline Ancho de pulso & $<5 \mathrm{~ns}$ & $<5 \mathrm{~ns}$ \\
\hline Fluencia & $7 \mathrm{~J} / \mathrm{cm}^{2}$ & $7 \mathrm{~J} / \mathrm{cm}^{2}$ \\
\hline Frecuencia & $8 \mathrm{~Hz}$ & $3 \mathrm{~Hz}$ \\
\hline Tamaño del diámetro de ablación & $35 \mu \mathrm{m}$ & $30 \mu \mathrm{m}$ \\
\hline Duración de la ablación & $21 \mathrm{~s}$ & $60 \mathrm{~s}$ \\
\hline Línea base & $15 \mathrm{~s}$ & $20 \mathrm{~s}$ \\
\hline Tiempo de limpieza entre ablaciones & $20 \mathrm{~s}$ & $15 \mathrm{~s}$ \\
\hline Gas de arrastre & $100 \%$ He en la celda y para transporte hacia la antorcha & $\begin{array}{l}100 \% \mathrm{He} \text {, con flujo adicional de mezcla Ar y y } \mathrm{N}_{2}(3 \mathrm{ml} / \\
\mathrm{min})\end{array}$ \\
\hline Flujo de gas de arrastre & 0,350 LPM & 0,750 LPM \\
\hline \multicolumn{3}{|c|}{ Instrumento ICP-MS } \\
\hline & Laboratorio SGC & Laboratorio Rennes \\
\hline Marca, modelo y tipo & Thermo Scientific, Element II & Agilent $7700 x$ \\
\hline Introducción de muestra & A través de tubos flexibles tipo tygon & A través de tubos convencionales \\
\hline Conos & $\mathrm{Ni}$ & $\mathrm{Ni}$ \\
\hline RF power & $1.200 \mathrm{~W}$ & $1.300 \mathrm{~W}$ \\
\hline Gas de arrastre & Ar $0,900 \mathrm{l} / \mathrm{min}$ & Ar $0,850 \mathrm{l} / \mathrm{min}$ \\
\hline Sistema de detección & Multiplicador de electrones Monocolector & Multiplicador de electrones Monocolector \\
\hline Masas medidas & ${ }^{202} \mathrm{Hg},{ }^{204}(\mathrm{Hg}+\mathrm{Pb}),{ }^{206} \mathrm{~Pb},{ }^{207} \mathrm{~Pb},{ }^{208} \mathrm{~Pb},{ }^{232} \mathrm{Th}$ y ${ }^{238} \mathrm{U}$ & ${ }^{202} \mathrm{Hg},{ }^{204}(\mathrm{Hg}+\mathrm{Pb}),{ }^{206} \mathrm{~Pb},{ }^{207} \mathrm{~Pb},{ }^{208} \mathrm{~Pb},{ }^{232} \mathrm{Th} y{ }^{238} \mathrm{U}$ \\
\hline Modo de detector & $\begin{array}{l}\text { Contador de pulsos, corrección por tiempo muerto } \\
\text { aplicada y modo análogo para cps }>4 \times 10^{6} \mathrm{cps}\end{array}$ & $\begin{array}{l}\text { Contador de pulsos, corrección por tiempo muerto } \\
\text { aplicada y modo análogo para cps }>10^{6} \mathrm{cps}\end{array}$ \\
\hline Tiempo de integración por pico & $10-30 \mathrm{~ms}$ & $10-30 \mathrm{~ms}$ \\
\hline \multicolumn{3}{|c|}{ Procesamiento de datos } \\
\hline & LG - SGC & Laboratorio Rennes \\
\hline Blanco & $\begin{array}{l}20 \text { s de señal, correspondiente al gas puro antes de la } \\
\text { ablación }\end{array}$ & $20 \mathrm{~s}$ antes del pico \\
\hline Estrategia de calibración & $\begin{array}{l}\text { Plesovice usado como material de referencia primario, } \\
\text { FC } 5 \text { Duluth y Mount Dromedary usados como } \\
\text { materiales de referencia secundario y terciario para } \\
\text { monitorear y comparar los resultados obtenidos con } \\
\text { MR primario }\end{array}$ & $\begin{array}{l}\text { GJ } 1 \text { usado como material de referencia primario, } \\
\text { Plesovice usado como material de referencia secundario }\end{array}$ \\
\hline Materiales de referencia (circones) & $\begin{array}{l}\text { Plesovice (edad 337,13 } \pm 0,37 \text { Ma, Sláma et al., 2008), } \\
\text { FC-5 Duluth (edad 1.099 } 0 \text { 0,6 Ma, Paces y Miller, 1993) } \\
\text { y Mount Dromedary (edad 99,12 } \pm 0,14 \text { Ma, Renne et } \\
\text { al., 1998) }\end{array}$ & $\begin{array}{l}\text { GJ } 1 \text { (edad 607,7 } \pm 0,67 \mathrm{Ma} \text {, (Jackson, Pearson, Griffin } \\
\text { y Belousova, 2004), Plesovice (edad } 337,13 \pm 0,37 \mathrm{Ma} \text {, } \\
\text { Sláma et al., 2008) }\end{array}$ \\
\hline $\begin{array}{l}\text { Programa de procesamiento de datos/corrección de } \\
\text { fraccionamiento de elementos inducido por láser (LIEF) }\end{array}$ & $\begin{array}{l}\text { lolite } v 2.5 \text { para normalización, propagación de la } \\
\text { incertidumbre y cálculo de la edad. Se asume que la } \\
\text { corrección LIEF del material de referencia es idéntico } \\
\text { para las muestras y se realiza mediante una ecuación } \\
\text { del tipo y }=a+b . e^{-c x}\end{array}$ & $\begin{array}{l}\text { Hoja de cálculo propia para normalización, propagación } \\
\text { de la incertidumbre y cálculo de la edad. Se asume que } \\
\text { la corrección LIEF del material de referencia es idéntico } \\
\text { para las muestras }\end{array}$ \\
\hline Corrección de Pb común, composición e incertidumbre & $\begin{array}{l}\text { No se aplicó corrección de Pb común a los datos } \\
\text { obtenidos }\end{array}$ & $\begin{array}{l}\text { No se aplicó corrección de Pb común a los datos } \\
\text { obtenidos }\end{array}$ \\
\hline Nivel de incertidumbre y propagación & $\begin{array}{l}\text { Edades calculadas a } 2 \text { desviaciones estándar, } \\
\text { propagación realizada por adición cuadrática }\end{array}$ & $\begin{array}{l}\text { Edades calculadas a } 2 \text { desviaciones estándar, } \\
\text { propagación realizada por adición cuadrática }\end{array}$ \\
\hline Control de calidad/validación & $\begin{array}{l}\text { FC } 5 \text { Duluth edad promedio }{ }^{206} \mathrm{~Pb} / 238 \mathrm{U}=1103,4 \pm 3,9 \\
(2 \mathrm{~s}, \mathrm{MSWD}=0,53, \mathrm{n}=25), \text { Mount Dromedary, edad } \\
\text { promedio }{ }^{206} \mathrm{~Pb} / 238 \mathrm{U}=100,10 \pm 0,61 \mathrm{Ma}(2 \mathrm{~s}, \mathrm{MSWD}= \\
0,78, \mathrm{n}=20)\end{array}$ & $\begin{array}{l}\text { Plesovice: edad de concordia }=336,5 \pm 5,5 \mathrm{Ma}(\mathrm{N}=2 ; \\
\mathrm{MSWD}=0,18 ; \text {; probability }=0,91)\end{array}$ \\
\hline
\end{tabular}

Fuente: autores 


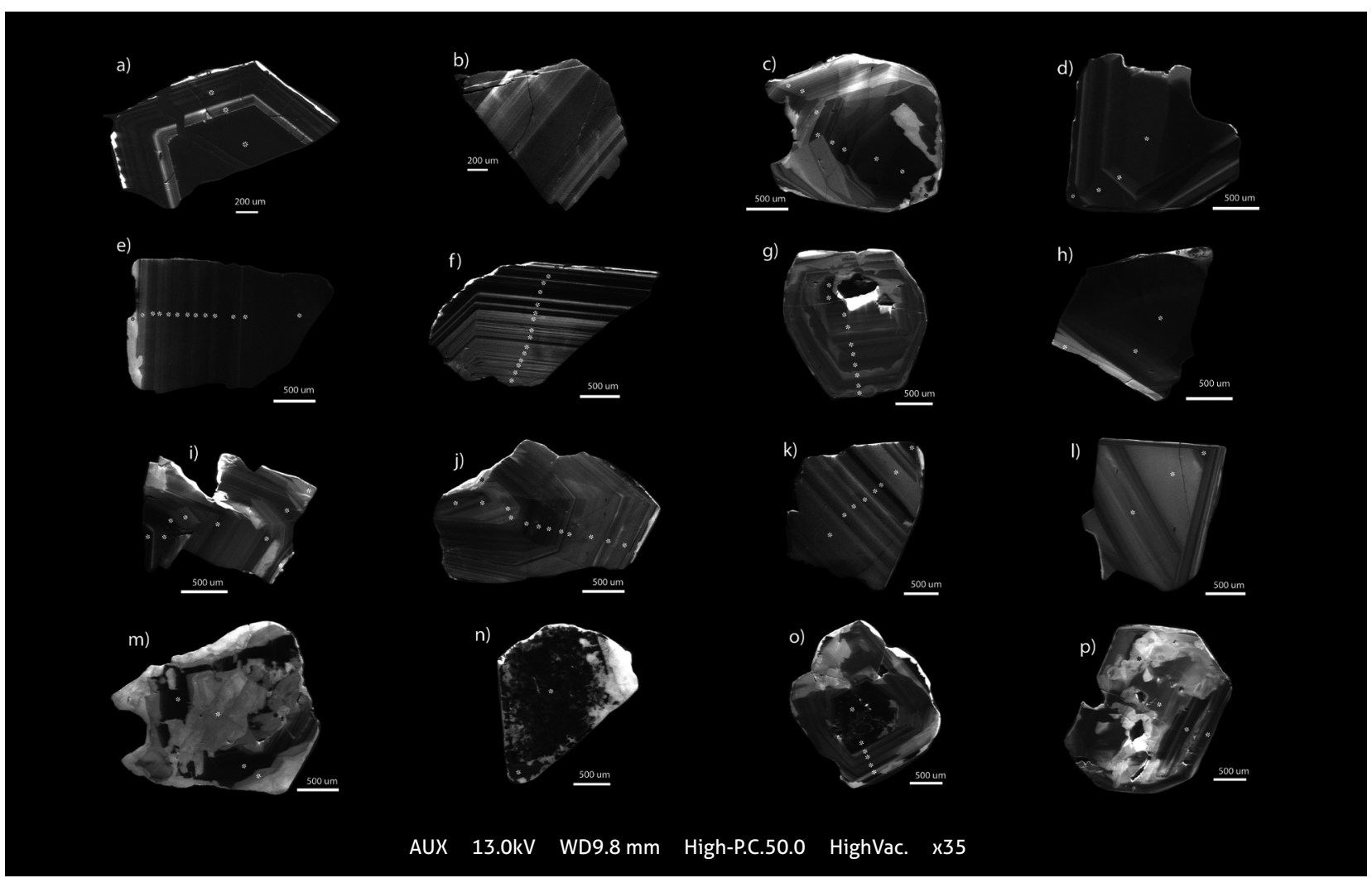

Figura 4. Imágenes de catodoluminiscencia de los circones más representativos. a - p: circones sienita nefelínica del Jordán, San José del Guaviare; $(\mathrm{m}-\mathrm{p})$ circones con daño radiogénico.

Fuente: autores

\section{Resultados}

\subsection{Laboratorio del Servicio Geológico Colombiano}

Los resultados de las mediciones de las relaciones isotópicas ${ }^{206} \mathrm{~Pb},{ }^{207} \mathrm{~Pb},{ }^{235} \mathrm{U},{ }^{238} \mathrm{U}$ y de $\mathrm{U}_{\text {total }}$ y Th con las correspondientes desviaciones estándar $2 \mathrm{~s}$ de los circones analizados en la tabla 2 solamente toman en cuenta edades concordantes calculadas por debajo de un umbral de discordia definido $<$ al $5 \%$.

La discordancia de datos $\mathrm{U} / \mathrm{Pb}$ en circones es definida como la no coincidencia de datos isotópicos derivados de dos o más sistemas isotópicos (Wetherill, 1956). Se debe generalmente a la migración de $\mathrm{Pb}$ (y en menor medida de $U$ ) y se la cuantifica usando relaciones de edades o de ${ }^{206} \mathrm{~Pb} /{ }^{238} \mathrm{U}$ y ${ }^{207} \mathrm{~Pb} /{ }^{206} \mathrm{~Pb}$ (Tera y Wasserburg, 1972) o de ${ }^{206} \mathrm{~Pb} /{ }^{238} \mathrm{U}$ y ${ }^{207} \mathrm{~Pb} /{ }^{235} \mathrm{U}$ (Wetherill, 1956). La concordia es la curva en la que las edades derivadas de los dos sistemas coinciden. Las bajas concentraciones, sobre todo de ${ }^{235} \mathrm{U}$ y de su isótopo hijo ${ }^{207} \mathrm{~Pb}$, causan que ${ }^{235} \mathrm{U}$ a menudo no se mida, y se calcule ${ }^{235} \mathrm{U}$ usando la relación ${ }^{238} \mathrm{U} / 235 \mathrm{U} \sim$ 137,88 para estimar principalmente la discordancia del análisis (Spencer, Kirkland y Taylor, 2016). Por eso en análisis de circones se emplean efectivamente dos edades isotópicas $\left({ }^{206} \mathrm{~Pb} /{ }^{238} \mathrm{U}\right.$ y $\left.{ }^{207} \mathrm{~Pb} /{ }^{206} \mathrm{~Pb}\right)$, de las cuales se escoge la "mejor edad". Normalmente se usa la edad ${ }^{207} \mathrm{~Pb} /{ }^{206} \mathrm{~Pb}$ para circones que sobrepasen la edad de 1,2 Ga, y las edades ${ }^{206} \mathrm{~Pb} /{ }^{238} \mathrm{U}$ para circones de origen más reciente (Gehrels, Valencia y Ruiz, 2008). El análisis de ${ }^{207} \mathrm{~Pb}$ es más impreciso, con ratas de conteo de casi una magnitud menor que el ${ }^{206} \mathrm{~Pb}$, lo que resulta en mayor imprecisión de las edades ${ }^{207} \mathrm{~Pb} /{ }^{206} \mathrm{~Pb}$ de circones de menos de $1,2 \mathrm{Ga}$.

Una de las ventajas de usar diagramas concordia U/ $\mathrm{Pb}$ en vez de isócronas es que inicialmente se puede dejar de lado el cálculo de plomo inicial residual no radiogénico $\left({ }^{204} \mathrm{~Pb}\right)$ y de procesos radiogénicos anteriores $\left({ }^{207} \mathrm{~Pb}_{0}\right.$ y ${ }^{206} \mathrm{~Pb}_{0}$ ), así que solamente entra plomo radiogénico después del cierre $\left({ }^{207} \mathrm{~Pb}^{*} \mathrm{y}^{206} \mathrm{~Pb}^{*}\right)$ en la ecuación para calcular la edad t, por ejemplo: ${ }^{206} \mathrm{~Pb}^{*} / 238 \mathrm{U}=\left(\mathrm{e}^{\lambda}{ }_{238}{ }^{\mathrm{t}}-1\right)$, que se expresa en el grado de la curvatura concordia. 
Tabla 3. Relaciones isotópicas medidas para ${ }^{206} \mathrm{~Pb},{ }^{207} \mathrm{~Pb},{ }^{235} \mathrm{U},{ }^{238} \mathrm{U}$ y de Utotal y Th y edades calculadas de los circones de la Sienita Nefelinica de San José del Guaviare, analizados en el SGC

\begin{tabular}{|c|c|c|c|c|c|c|c|c|c|c|c|c|c|}
\hline $\begin{array}{r}6 \text { de febrero } \\
\text { Datación U }\end{array}$ & $\begin{array}{l}\text { de } 2018 \\
\text { /Pb, Sen } \\
\text { Colomb }\end{array}$ & $\begin{array}{l}\text { 8, Laborat } \\
\text { vicio Geol } \\
\text { iano }\end{array}$ & $\begin{array}{l}\text { o de } \\
\text { ico } \\
\end{array}$ & & atos para & $\begin{array}{l}\text { diagrama } \\
\text { serburg }\end{array}$ & & & atos pa & lagrama & Wetherill & & \\
\hline $\begin{array}{l}\text { Identifi- } \\
\text { cador } \\
\text { (a) }\end{array}$ & $\begin{array}{l}\text { F206c } \\
\text { (b) }\end{array}$ & $\begin{array}{c}\text { Concen- } \\
\text { tración } \\
\sim \cup \text { ug. } \\
\mathrm{g}^{-1} \\
\text { (c) }\end{array}$ & $\begin{array}{l}\text { Th/U } \\
\text { (d) }\end{array}$ & $\begin{array}{c}206 \mathrm{~Pb} / 238 \mathrm{U} \\
\text { (e) }\end{array}$ & $\begin{array}{l}\text { \% Err. } \\
\text { (f) }\end{array}$ & $\begin{array}{c}{ }^{207} \mathrm{~Pb} / \\
{ }^{206} \mathrm{~Pb} \\
(\mathrm{e})\end{array}$ & $\begin{array}{l}\text { \% Err. } \\
\text { (f) }\end{array}$ & $\begin{array}{l}{ }^{207} \mathrm{~Pb} / \\
{ }^{235} \mathrm{U}(\mathrm{e})\end{array}$ & $\begin{array}{l}\text { \% Err. } \\
\text { (f) }\end{array}$ & $\begin{array}{l}{ }^{206} \mathrm{~Pb} / \\
{ }^{238} \mathrm{U}(\mathrm{e})\end{array}$ & $\begin{array}{l}\text { \% Err. } \\
\text { (f) }\end{array}$ & $\begin{array}{l}\text { Rho } \\
\text { (g) }\end{array}$ & $\begin{array}{l}\text { Edad } \\
{ }^{207} \mathrm{~Pb} / \\
{ }^{206} \mathrm{~Pb} \\
\mathrm{Ma}(\mathrm{h})\end{array}$ \\
\hline SNG-1-51 & N.R. & 28,26 & 0,60 & 10,225 & 0,00154 & 0,0609 & 0,0015 & 0,8120 & 0,0230 & 0,09780 & 0,00154 & 0,033 & 630,0 \\
\hline SNG-1-58 & N.R. & 45,15 & 0,82 & 10,215 & 0,00196 & 0,0611 & 0,0013 & 0,8220 & 0,0170 & 0,09790 & 0,00196 & 0,055 & \\
\hline SNG-1-116 & N.R. & 21,06 & 0,49 & 10,215 & 0,00224 & 0,0597 & 0,0016 & 0,8160 & 0,0250 & 0,09790 & 0,00224 & 0,037 & 600,0 \\
\hline SNG-1-148 & N.R. & 61,20 & 0,60 & 10,215 & 0,00196 & 0,0607 & 0,0011 & 0,8090 & 0,0170 & 0,09790 & 0,00196 & 0,035 & 624,0 \\
\hline SNG-1-162 & N.R. & 35,81 & 0,43 & 10,215 & & 0,0595 & 0,0013 & 0,8060 & 0,0200 & 0,09790 & 0,00140 & 0,054 & 579,0 \\
\hline SNG-1-178 & N.R. & 14,94 & 0,30 & 10,204 & 0,00224 & 0,0597 & 0,0020 & 0,8060 & 0,0270 & 0,09800 & 0,00224 & 0,060 & 612,0 \\
\hline SNG-1-128 & N.R. & 79,00 & 0,40 & 10,204 & & 0,0601 & 0,0009 & 0,8080 & 0,0120 & & & 0,075 & 604,0 \\
\hline SNG-1-146 & N.R. & 88,80 & 0,62 & 10,204 & 0,00154 & 0,0602 & 0,0009 & 0,8170 & 0,0140 & 0,09800 & 0,00154 & 0,049 & 616,0 \\
\hline SNG-1-140 & N.R. & 65,70 & 0,56 & 10,194 & 0,00140 & 0,0601 & 0,0014 & 0,8050 & 0,0190 & 0,09810 & 0,00140 & 0,042 & 611,0 \\
\hline SNG-1-19 & N.R. & 15,06 & 0,3 & 10,194 & & 0,0598 & 0,0020 & 0,8160 & 0,0260 & 0,09810 & 0,00182 & 0,037 & 584,0 \\
\hline SNG-1-1 & N.R. & 26,39 & 0,4 & 10,194 & & 0,0596 & 0,0010 & 0,8210 & 0,0170 & 0,09810 & & 0,059 & 594,0 \\
\hline SNG-1-117 & N.R. & 45,05 & 0,68 & 10,183 & 0,00238 & 0,0594 & 0,0011 & 0,8030 & 0,0200 & 0,09820 & 0,00238 & 0,043 & 578,0 \\
\hline SNG-1-1 & N.R. & 35,34 & 0,24 & 10,183 & & 0,0595 & 0,0012 & 0,8030 & 0,0130 & & & 0,022 & 582,0 \\
\hline SNG-1-1 & N.R. & 50,2 & 0,5 & 10,176 & & 0,0595 & 0,0009 & 0,8040 & 0,0120 & 0,09827 & 0,00118 & 0,057 & 583,0 \\
\hline SNG-1-189 & N.R. & 29,19 & 0,95 & 10,171 & & 0,0596 & 0,0013 & 0,8210 & 0,0150 & 0,09832 & & 0,078 & 584,0 \\
\hline SNG-1- & N.R. & 118,89 & 0,7 & 10,16 & & 0,0605 & 0,00 & 0,8160 & 0,0140 & 0,09842 & 0,00104 & 0,033 & 619,0 \\
\hline SNG-1-1 & N.R. & 61,61 & 0,60 & 10,163 & & 0,0602 & & 0,8250 & & 0,09840 & & 0,050 & 615,0 \\
\hline SNG-1-1 & N.R. & 65,9 & 0,6 & 10,1 & & 0,0611 & 0,0012 & 0,8270 & 0,01 & 0,09 & 0,00 & 0,066 & 649 \\
\hline SNG-1-1 & N.R. & 12,2 & 1,4 & & & & & 0,8250 & & & & 0,052 & \\
\hline SNG-1-61 & N.R. & 64,60 & 0,69 & 10,152 & 0,00182 & 0,0595 & 0,0012 & 0,8010 & 0,0160 & 0,09850 & 0,001 & 0,043 & 580,0 \\
\hline SNG-1-7 & N.R. & 354, & 2,80 & & & & & 0,8141 & & & & 0,064 & \\
\hline SNG-1-157 & N.R. & 43,90 & 0,59 & 10,150 & 0,0 & 0,0608 & 0,0017 & 0,8210 & 0,0200 & 0,098 & 0,00 & 0,035 & 622,0 \\
\hline SNG-1-11 & N.R. & 108,30 & 0,93 & 10,148 & & 0,0606 & 0000 & 0,8210 & & & & 0,062 & 624,0 \\
\hline SNG- & N.R. & 59,0 & 0,6 & 10,1 & & 0,060 & 0,00 & 8252 & 0,00 & 0,098 & 0,00 & 0,059 & 633 \\
\hline SNG-1 & N.R. & 16,3 & 0,47 & & & 0,060 & 0,00 & 0,8180 & & & & 0,076 & 619 \\
\hline SNG-1- & N.R. & 31,51 & 0,4 & 10,14 & 0,001 & 0,0607 & 0,0015 & 0,8310 & 0,0230 & 0,09860 & 96 & 0,070 & 668,0 \\
\hline SNG-1-144 & N.R. & 259,30 & 1,09 & & & 0,05 & & 0,8050 & & & & 0,046 & \\
\hline SNG-1-12 & N.R. & 54,16 & 0,86 & 10,1 & 0,00 & 0,06 & 0,0011 & 0,8270 & 0,0150 & 0,09862 & 04 & 0,070 & 624 \\
\hline SNG-1-15 & N.R. & 64,02 & & & & & & 0,8235 & & & & 0,050 & \\
\hline SNG-1- & N.R. & 84,0 & 0,56 & 10,1 & 0,001 & 0,059 & 0,0010 & 0,8190 & 0,0130 & 0,09860 & 0,00 & 0,099 & 592,0 \\
\hline SNG-1-45 & N.R. & 635,00 & 2,56 & & & 0,060 & 0,00 & 0,8250 & 0,01 & & & 0,053 & 620,0 \\
\hline SNG-1 & N.R. & 575, & 2,05 & & & 0,06 & 0,00 & 0,8220 & & 0,098 & 0,00 & 0,049 & \\
\hline SNG-1-139 & N.R. & 118,20 & 0,75 & & & 0,0603 & 0,00 & 0,8130 & 0,01 & 0,09867 & & 0,054 & 61 \\
\hline SNG-1-145 & N.R. & 145,2 & 0,81 & 1 & & 0,06 & 0,00 & 0,8130 & 0,0120 & 0,09870 & 68 & 0,049 & 607 \\
\hline SNG-1-16 & N.R. & 48,59 & & & & 0,05 & 0,00 & 0,8140 & 0,01 & & & 0,053 & \\
\hline SNG-1-1 & N.R. & 98,80 & 0,74 & & & 0,06 & 0,00 & 0,8157 & 0,00 & 70 & & 0,066 & 61 \\
\hline SNG-1-175 & N.R. & 98,20 & 1,37 & 10,132 & 0,001 & 0,0607 & 0,00 & 0,8240 & 0,0160 & 0,09870 & 0,00 & 0,066 & 625,0 \\
\hline SNG-1-99 & N.R. & 12,87 & 5,3 & & & 0,0607 & 0,0019 & 0,8280 & 0,0230 & 0,09870 & 0,00 & 0,041 & 620,0 \\
\hline SNG-1-114 & N.R. & 66,10 & 0,69 & 0,132 & 0,00196 & 0,0610 & 0,0011 & 0,8200 & 0,0180 & 0,09870 & 0,00196 & 0,045 & 635,0 \\
\hline SNG-1-142 & N.R. & 139,80 & 0,87 & 0,128 & & 0,0610 & 0,0010 & 0,8230 & 0,0110 & 0,09874 & 0,00108 & 0,041 & 637,0 \\
\hline SNG-1-141 & N.R. & 78,30 & 0,63 & 0,121 & 0,00168 & 0,0606 & 0,0011 & 0,8190 & 0,0160 & 0,09880 & 0,00168 & 0,099 & 622,0 \\
\hline SNG-1-156 & N.R. & 124,70 & & 10,119 & 0,00120 & 0,0601 & 0,0007 & 0,8211 & 0,0091 & 0,09882 & 0,00120 & 0,058 & 607,0 \\
\hline SNG-1-172 & N.R. & 237,30 & 1,57 & & & 0,0600 & 0,0008 & 0,8192 & 0,0099 & & & 0,035 & 602,0 \\
\hline SNG-1-187 & N.R. & & 36,34 & & & 0,0 & 0,0014 & 0,8150 & 0,0240 & 09880 & 96 & 0,025 & 596,0 \\
\hline SNG-1-70 & N.R. & 76,60 & 0,57 & 114 & & 0,0607 & 0,0012 & 0,8180 & 0,0150 & 0,09887 & 0,00 & 0,076 & 632,0 \\
\hline SNG-1-5 & N.R. & 43,48 & 6 & & & 0,0604 & 0,0014 & 0,8210 & 0,0190 & 0,09890 & & 0,031 & 612,0 \\
\hline SNG-1-167 & N.R. & 520,30 & 2,55 & & 1140 & 0,0597 & 0,0004 & 0,8138 & 0,0071 & 0,09900 & & 0,070 & 597,0 \\
\hline SNG-1-192 & N.R. & 50,44 & 0,54 & & 0,00140 & 0,0607 & 0,0011 & 0,8280 & 0,0120 & 0,09900 & 0,00 & 0,048 & 625,0 \\
\hline SNG-1-182 & N.R. & & & & & & & 0,8190 & & & & 0,070 & \\
\hline SNG-1-155 & N.R. & 10,49 & 0,34 & 1 & 0,00182 & 0,0604 & 0,0027 & 0,8360 & 0,0370 & 0,09900 & 82 & 0,044 & 598,0 \\
\hline SNG-1-56 & N.R. & 127,00 & & & & & & 0,8250 & & & & 0,058 & \\
\hline SNG-1-91 & N.R. & 38,09 & 3,01 & 0,096 & 130 & 0,0597 & 0,0014 & 0,8130 & 0,0210 & 0,09905 & & 0,070 & 595,0 \\
\hline SNG-1-102 & N.R. & 121,10 & 0,81 & & & 0,0604 & 0,0009 & 0,8230 & 0,0120 & 0,09900 & & 0,060 & 623,0 \\
\hline SNG-1-19 & N.R. & & & & & & & 0,8340 & 0,0220 & 0,09910 & & 0,065 & 652,0 \\
\hline SNG-1-200 & N.R. & & & & & & 0,0 & 0,8260 & 0,0130 & 0,09910 & & 0,040 & 621,0 \\
\hline SNG-1-86 & N.R. & 175,60 & & & & 0,0609 & 0,0009 & 0,8240 & 0,0140 & 09917 & & 0,057 & 638,0 \\
\hline SNG-1-111 & N.R. & & & & & & & & & & & & \\
\hline SNG-1-174 & N.R. & 08,60 & 1,18 & 0,081 & 0154 & 0,0597 & 0,0008 & 0,8120 & 0,0110 & 0,09920 & 0,00 & 0,056 & 596,0 \\
\hline SNG-1-55 & N.R. & & & & & & & 0,8260 & & & & 0,051 & \\
\hline SNG-1 & N.R. & & & & & & & 8180 & & & & 0,07 & 598 \\
\hline SNG- & N.R. & & & & & & & 0,8280 & & & & & \\
\hline SNG-1-131 & N.R. & & & & & & & 8240 & & & & 0,094 & 619 \\
\hline & N.R. & & & & & & & & & & & & \\
\hline NG-1-112 & N.R. & & & & & & & 8270 & & & & 0,062 & 61 \\
\hline & N.R. & & & & & & & & & & & & \\
\hline SNG & N.R. & 10 & & & & 0601 & 0,00 & 18230 & 10 & 09930 & & 0,065 & 603,0 \\
\hline & N.R. & & & & & & & & & & & & \\
\hline SNC & N.R. & & & & & & & 8249 & & & & 0,0 & \\
\hline & N.R. & & & & & & & & & & & & \\
\hline SNG-1-132 & N.R. & & & & & 0599 & & 8240 & & 09970 & & 0,034 & 597,0 \\
\hline & N.R. & & & & & & & & & & & & \\
\hline SNG & N.R. & & & & & & & 50 & & & & 0,085 & 62 \\
\hline & N.R. & & & & & & & & & & & 0,092 & \\
\hline 5 & N.R. & & & & & 0606 & 0 & 8220 & & & & 0,045 & 62 \\
\hline & N.R. & 154,50 & & & & 1,0611 & 0,0007 & 0,8410 & 0,0120 & 0,09990 & & 0,070 & 643,0 \\
\hline & & & & & & & & & & & & & \\
\hline SNG-1-22 & N.R. & 223,80 & & 013 & 00118 & 0609 & 0,0004 & 0,8411 & 0,0080 & 0,09987 & 0,00118 & 0,044 & 634,0 \\
\hline & & & & & & & & & & & & 0,074 & \\
\hline SNG-1-2 & N.R. & 291,00 & 3, & 010 & 0136 & 0600 & 0,0006 & 0,8288 & 0,0074 & 09990 & 0,00136 & 0,048 & 604,0 \\
\hline & & & & & & & & & & & & & \\
\hline SNG-1-48 & N.R. & 326,70 & 1,39 & 0,010 & 00140 & 0,0602 & 0,0006 & 0,8220 & 0,0100 & 0,09990 & 0,00140 & 0,070 & 619,0 \\
\hline & N.R. & & & & & & & & & & & & \\
\hline SNG-1-169 & N.R. & 20,57 & 0,65 & 10,010 & 0,00210 & 0,0605 & 0,0017 & 0,8370 & 0,0240 & 0,09990 & 0,00210 & 0,057 & 623,0 \\
\hline
\end{tabular}




\begin{tabular}{|c|c|c|c|c|c|c|c|c|c|c|c|c|c|c|c|c|c|c|c|c|c|c|}
\hline \multicolumn{4}{|c|}{$\begin{array}{c}6 \text { de febrero de 2018, Laboratorio de } \\
\text { Datación U/Pb, Servicio Geológico } \\
\text { Colombiano }\end{array}$} & \multicolumn{4}{|c|}{$\begin{array}{l}\text { Datos para diagrama } \\
\text { Tera-Wasserburg }\end{array}$} & \multicolumn{5}{|c|}{ Datos para diagrama Wetherill } & \multicolumn{9}{|c|}{ Edades obtenidas } & \multirow[b]{2}{*}{$\begin{array}{l}\text { \% Dis- } \\
\text { cordia. } \\
\text { (k) }\end{array}$} \\
\hline $\begin{array}{l}\text { Identifi- } \\
\text { cador } \\
\text { (a) }\end{array}$ & $\begin{array}{l}\text { F206c } \\
\text { (b) }\end{array}$ & 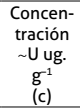 & $\begin{array}{l}\mathrm{Th} / \mathrm{U} \\
\text { (d) }\end{array}$ & $\begin{array}{c}206 \mathrm{~Pb} / 238 \mathrm{U} \\
\text { (e) }\end{array}$ & $\begin{array}{l}\text { \% Err. } \\
\text { (f) }\end{array}$ & $\begin{array}{l}{ }^{207} \mathrm{~Pb} / \\
{ }^{206} \mathrm{~Pb} \\
(\mathrm{e})\end{array}$ & $\begin{array}{c}\text { \% Err. } \\
\text { (f) }\end{array}$ & $\begin{array}{l}207 \mathrm{~Pb} / \\
235 \mathrm{U}(\mathrm{e})\end{array}$ & $\begin{array}{l}\text { \% Err. } \\
\text { (f) }\end{array}$ & $\begin{array}{l}{ }^{206 \mathrm{~Pb} /} \\
{ }^{238} \mathrm{U}(\mathrm{e})\end{array}$ & $\begin{array}{l}\text { \% Err. } \\
\text { (f) }\end{array}$ & $\begin{array}{l}\text { Rho } \\
\text { (g) }\end{array}$ & $\begin{array}{c}\text { Edad } \\
207 \mathrm{~Pb} / \\
206 \mathrm{~Pb} \\
\mathrm{Ma}(\mathrm{h})\end{array}$ & $\begin{array}{l}2 \text { sabs } \\
\text { (i) }\end{array}$ & $\begin{array}{c}2 \mathrm{~s} \\
\text { abs } \\
\text { prop. (j) }\end{array}$ & $\begin{array}{c}\text { Edad } \\
{ }^{206 \mathrm{~Pb} /} \\
238 \mathrm{U} \\
\text { (h) }\end{array}$ & $\begin{array}{l}2 \mathrm{~s} \\
\text { abs } \\
\text { (i) }\end{array}$ & $\begin{array}{l}2 \mathrm{~s} \\
\text { abs } \\
\text { prop. } \\
\text { (j) }\end{array}$ & $\begin{array}{c}\text { Edad } \\
207 \mathrm{~Pb} / \\
235 \mathrm{U} \\
\text { (h) }\end{array}$ & $\begin{array}{c}2 \mathrm{~s} \text { abs } \\
\text { (i) }\end{array}$ & $\underset{\text { prop. (j) }}{2 \mathrm{~s} \text { abs }}$ & \\
\hline SNG-1-35 & N.R. & 64,80 & 1,65 & & & 00607 & & 0,8370 & & & & & 618,0 & & & & 11,0 & 13,0 & & 9,3 & 12,0 & 0,2 \\
\hline SNG-1-38 & N.R. & 77,00 & 2,65 & 10,000 & 0,00154 & 0,0597 & 0,0010 & 0,8320 & 0,0160 & 0,10000 & 0,00154 & 0,060 & 591,0 & 35 & 39 & & 6,5 & 9,9 & 4,2 & 8,8 & 11,0 & $-0,1$ \\
\hline SNG-1-173 & N.R. & 247,30 & & & & 0,0607 & 0,0006 & 0,8353 & 0,0097 & 0,1000 & & 0,070 & 627,0 & 21 & 28 & & 4,9 & 8,9 & 16,4 & 5,4 & 9,1 & $-1,0$ \\
\hline SNG-1-50 & N.R. & 80,70 & 0,73 & 10,000 & 0,00210 & 0,0610 & 0,0009 & 0,8400 & 0,0150 & 0,10000 & 0,00210 & 0,033 & 637,0 & 33 & 38,0 & 61 & 8,9 & 12,0 & 620,5 & 7,6 & 10,0 & 0,4 \\
\hline SNG-1-7 & & 125,90 & & & & 0,06 & 然 & 0,8220 & & & & & 605 & 30 & & & 7 & 10,0 & 3,9 & 6,6 & 9,8 & 0,6 \\
\hline SNG-1-103 & N.R. & 296,20 & 1,39 & 9,991 & 0,00125 & 0,0599 & 0,0006 & 0,8230 & 0,0110 & 0,10009 & 0,00125 & 0,037 & 597,0 & 21,0 & 28,0 & 614 & 5,2 & 9,1 & 609,5 & 6,2 & 9,6 & $-0,7$ \\
\hline SNG-1-202 & N.R. & & & & & 0,0606 & 0,0009 & 0,8350 & 0,0120 & 0,10010 & 0,00154 & 0,035 & 621,0 & 32 & 36 & 615 & 6,7 & 10,0 & 616,0 & 6,7 & 10,0 & 0,3 \\
\hline SNG-1-76 & N.R. & 25,50 & 0,71 & 9,980 & 0,00168 & 0,0610 & 0,0008 & 0,8320 & 0,0140 & 0,10020 & 0,00168 & 0,054 & 643 & 27,0 & 32,0 & 615 & 6 & 10,0 & 614,6 & 7,6 & 11,0 & 0,8 \\
\hline & & 21,20 & & 9,980 & 0,00140 & 0,0605 & 0,0006 & 0,8220 & 0,0100 & 0,10020 & 0,00140 & 0,060 & 620 & 20 & 27,0 & 615 & 5,9 & 9,5 & 609,2 & 5,7 & 9,2 & $-1,0$ \\
\hline SNG-1-202 & N.R. & 28,26 & 0,60 & 10,225 & 0,00154 & 0,0609 & 0,0015 & 0,8120 & 0,0230 & 0,09780 & 0,00154 & 0,075 & 630,0 & 52,0 & 55,0 & 601,6 & 6,3 & 9,6 & 603,0 & 13,0 & 15,0 & $-1,0$ \\
\hline SNG-1-76 & & & & & & & & 0,8220 & 0,0170 & 0,09790 & & 0,049 & 638,0 & 47,0 & 50,0 & 602,0 & 8,1 & 11,0 & 608,7 & 9,5 & 12,0 & $-1,0$ \\
\hline SNG-1-79 & N.R. & 21,06 & 0,49 & 10,215 & 0,00224 & 0,0597 & 0,0016 & 0,8160 & 0,0250 & 0,09790 & 0,00224 & 0,042 & 600,0 & 62,0 & 65,0 & 602,1 & 9,2 & 12,0 & 608,0 & 15,0 & 17,0 & 0,6 \\
\hline SNG-1-202 & N.R. & 61,20 & 0,60 & 10,215 & 0,00196 & 0,0607 & 0,0011 & 0,8090 & 0,0170 & 0,09790 & 0,00196 & 0,037 & 624,0 & 38,0 & 42,0 & 602,1 & 8,3 & 11,0 & 603,4 & 9,2 & 11,0 & 0,1 \\
\hline
\end{tabular}

N.R.: no se realizó corrección por Pb común.

a) SNG-1-xx, xx corresponde al número de ablación.

b) Corrección $\mathrm{Pb}$ común no aplicada.

c) Concentración aproximada reportada por el programa Iolite v2.5.

d) Relación ${ }^{232} \mathrm{Th} /{ }^{238} \mathrm{U}$ calculada a partir de las intensidades obtenidas.

e) Relaciones elementales calculadas a partir de las intensidades obtenidas para cada isótopo.

f) Errores calculados a 2 sigma y propagados usando el algoritmo de Schmitz y Schoene (2007) y Crowley et al. (2007).

g) Parámetro Rho calculado como [(error interno $\left.\left.2 \mathrm{SE}^{206} \mathrm{~Pb} /{ }^{238} \mathrm{U}\right)^{2} \mathrm{x} 0,5\right] /\left(\right.$ error interno $2 \mathrm{SE}{ }^{206} \mathrm{~Pb} /{ }^{238} \mathrm{U}$ * error interno $2 \mathrm{SE}{ }^{207} \mathrm{~Pb} /{ }^{235} \mathrm{U}$ ) y utilizado para la concordancia de las edades. Schmitz y Schoene (2007)

h) Cálculos de edad basados en la constante de decaimiento radiactiva de Jaffey et al. (1971).

i) Errores como 2 sigma. La señal obtenida de cada isótopo es integrada y reducida mediante el programa Iolite v2.5 ${ }^{\circ}$ [10].

j) Errores como 2 sigma propagada. La señal obtenida de cada isótopo es integrada y reducida mediante el programa Iolite v2.5 ${ }^{\circledR}[10]$.

k) El \% Discordia = porcentaje de discordancia, corresponde a la diferencia porcentual entre la edad ${ }^{206} \mathrm{~Pb} /{ }^{238} \mathrm{U}$ y la edad ${ }^{207} \mathrm{~Pb} /{ }^{235} \mathrm{U}$.

Fuente: autores

La distribución de edades calculadas de 92 relaciones isotópicas de discordias $<5 \%$ con una curva de distribución normal sobrepuesta en la figura 5 muestra un rango de edades entre 600 y $616 \mathrm{Ma}$ y un promedio de $608 \mathrm{Ma}$, aunque con otra acumulación hacia $614 \mathrm{Ma}$ :

Las edades concordia, calculadas de 92 mediciones, en los diagramas de concordia ${ }^{207} \mathrm{~Pb} /{ }^{206} \mathrm{~Pb}$ vs. ${ }^{238} \mathrm{U} / 206 \mathrm{~Pb}$ (Tera y Wasserburg, 1975) de 608,8 $\pm 1,3 \mathrm{Ma}{ }^{206} \mathrm{~Pb} /{ }^{238} \mathrm{U}$ vs. ${ }^{207} \mathrm{~Pb} /{ }^{235} \mathrm{U}$ (Wetherill 1956) de $608,6 \pm 1,2 \mathrm{Ma}$ en figura 6 , solamente difieren levemente alrededor de la edad promedio de $608 \mathrm{Ma}$.

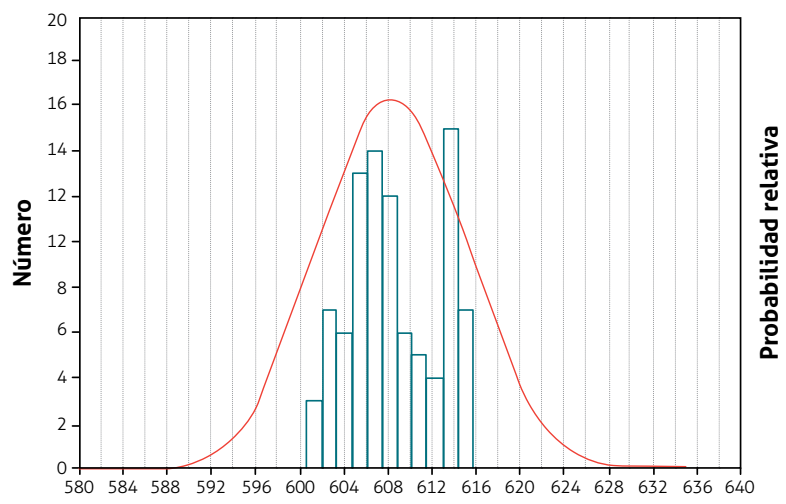

Figura 5. Histograma de edades correspondiente a la sienita. Fuente: autores
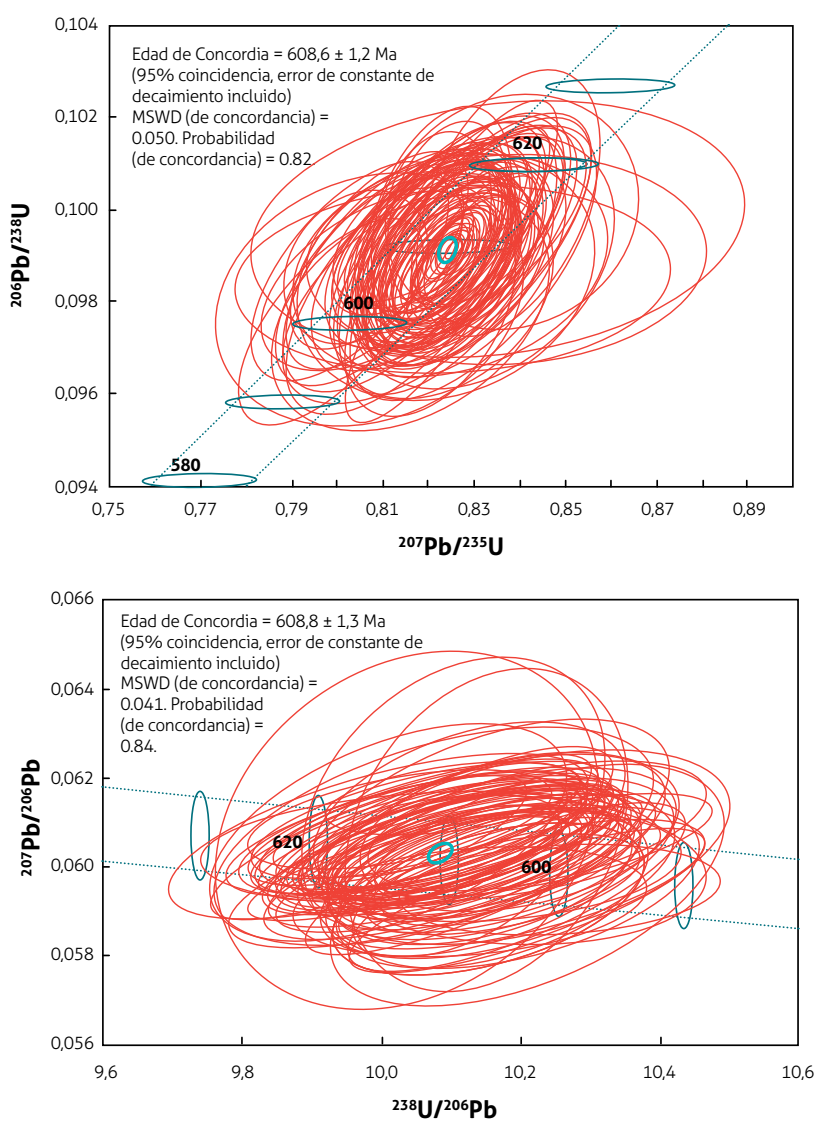

Figura 6. Edades de concordia correspondientes a la muestra de sienita. Arriba: diagrama Wetherill; abajo: Tera Wasserburg. Fuente: autores 
Las edades, sin descartar datos en el diagrama concordia de la figura 7, muestran adicionalmente una alta dispersión y datos discordantes que indican abundante pérdida de $\mathrm{Pb}$ en los anillos externos de cristales, con daño metamíctico y áreas con recristalización de $\sim 20 \mu \mathrm{m}$, que frecuentemente dan una respuesta baja en catodoluminiscencia (e.g., figuras $4 \mathrm{a}, 4 \mathrm{~m}$ y $4 \mathrm{o}$ ). En contraste, los análisis realizados en sectores no alterados que preservan la zonación oscilatoria intacta dieron siempre resultados concordantes, lo que son las zonas recomendadas para futuros análisis.

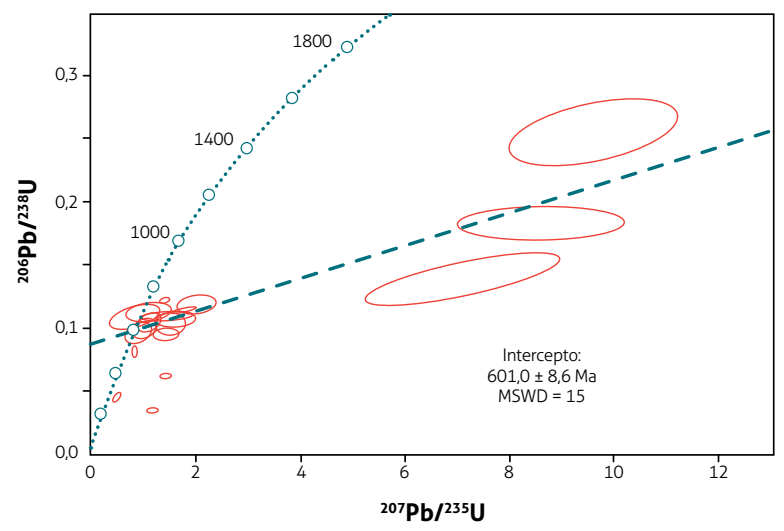

Figura 7. Diagrama de concordia Wetherill, obtenido en el SGC sin descartar datos, con un total de 202 análisis. Fuente: autores

La figura 7 muestra una alta dispersión y datos discordantes que indican fuerte perturbación en los anillos externos de cristales con daño metamíctico resultando en pérdida de $\mathrm{Pb}$ y áreas con recristalización de $\sim 20 \mu \mathrm{m}$, que frecuentemente dan una respuesta baja en catodoluminiscencia (e. g., figuras 4a, $4 \mathrm{~m}$ y 4o). Estos corresponden a los once datos más discordantes; en contraste, los análisis realizados en sectores no alterados que preservan la zonación oscilatoria intacta dieron siempre resultados concordantes. En este cálculo inicial se filtraron datos de borde de los cristales en contacto con la resina altamente discordantes y sectores con fracturas e inclusiones en las caras cristalinas (total, once datos), para un total de 191 ablaciones.

Las edades obtenidas de los materiales de referencia (figura 8) en el SGC fueron, para FC-5 Duluth, 1103,4 \pm 3,9 Ma (1.099 $\pm 0,6$ Ma según Paces y Miller, 1993), y para Mount Dromedary, 100,10 \pm 0,61 Ma $(99,12 \pm 0,14 \mathrm{Ma}$ según Renne et al., 1998).

\subsection{Laboratorio de la Universidad de Rennes}

Las veinticuatro ablaciones y mediciones de los circones de la sienita nefelínica realizadas en el Laboratorio LAICP-MS de la Universidad de Rennes dieron una edad promedio, en el diagrama Tera Wasserburg, de $602 \pm 3$ Ma ( $\pm 0,5 \%)$ (figura 9$)$. Todos los datos fueron concordantes debido a que se habían escogido ablaciones en puntos cercanos a los medidos previamente en el SGC, que brindaron los mejores resultados y evitaron, sobre todo, los anillos recristalizados y zonas con daño metamíctico. Para el estándar primario GJ-1 (GJ-1 607,7 \pm 0,67 Ma, según Jackson et al., 2004) se realizaron diez ablaciones y tres ablaciones sobre el material de referencia interno Plesovice dieron una edad de $336,5 \pm 5,5 \mathrm{Ma}$ $( \pm 1,63 \%)$. Todos los resultados son concordantes, incluso en sectores de cristales cuya zonación interna es compleja (figura $4 \mathrm{~m}-\mathrm{p}$ ).

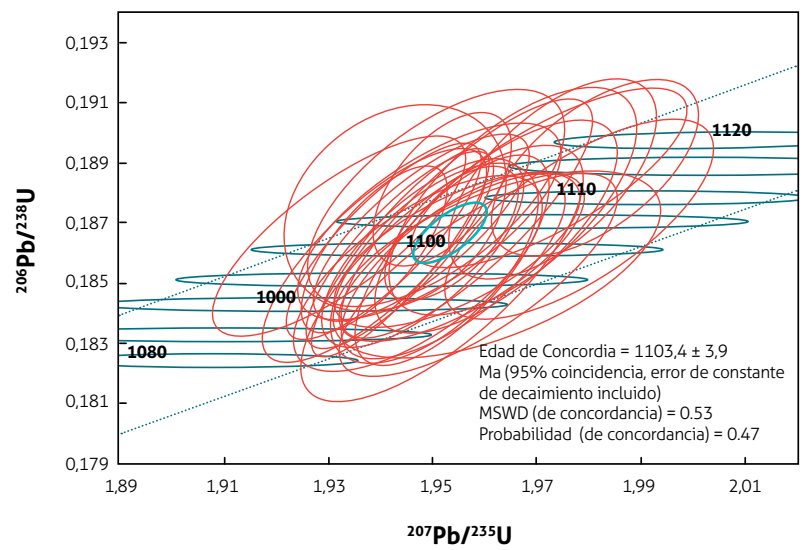

Figura 8. Edades de concordia correspondientes a los materiales de referencia, obtenidas en el SGC. Izquierda Mount Dromedary, derecha FC - 1 Duluth.

Fuente: autores 

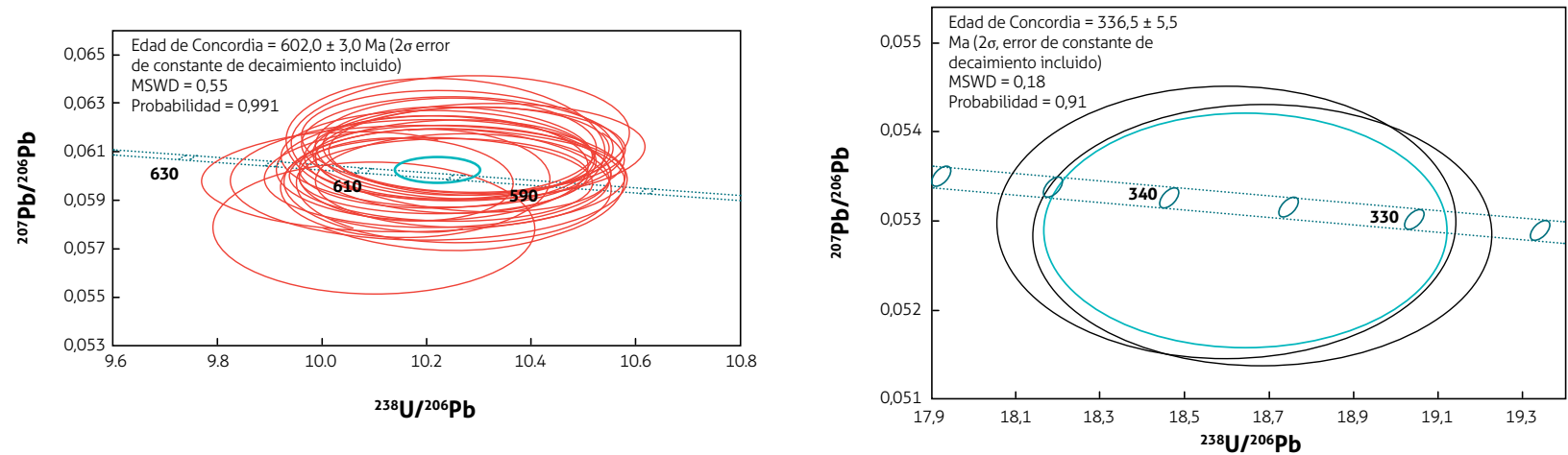

Figura 9. Laboratorio LA-ICPMS de Rennes. Izquierda, edades de concordia SNSJG calculadas en veinticuatro ablaciones, en el. Derecha material de referencia Plesovice.

Fuente: Laboratorio de la Universidad de Rennes, Francia

\subsection{Relación $T h / U$}

Las relaciones $\mathrm{Th} / \mathrm{U}$ pueden servir como criterio para determinar el origen de los circones, lo cual indica si estos minerales se han formado a partir de un fundido enriquecido en tierras raras, como el Th, o por recristalización subsólida en un ambiente empobrecido en estos elementos (Rubatto, 2002, 2017; Schaltegger, Fanning, Günther, Maurin, Schulmann y Gebauer, 1999). Aunque el valor Clarke de Th con 8,5 ppm es mayor que el de $\mathrm{U}$ con 1,7 ppm, la incorporación preferencial de $U$ en circones explicada en la introducción causa que una relación $\mathrm{Th} / \mathrm{U}>0,1$ indique un origen magmático de los circones.

Las relaciones $\mathrm{Th} / \mathrm{U}$ medidas tanto en el LG-SGC como en el Laboratorio de Geocronología de la Universidad de Rennes coinciden en términos analíticos y se encuentran dentro de la expectativa, de acuerdo con las edades obtenidas y el origen magmático de estos cristales, como puede observarse en la figura 10. Hay una alta dispersión de la relación $\mathrm{Th} / \mathrm{U}$, especialmente en los circones con poco $\mathrm{U}_{\text {total }}(5$ a $200 \mathrm{ppm})$, que tiende a inscribirse en una curva parabólica que se acerca al límite 6 de U/Th con el aumento de U.

La relación $\mathrm{Th} / \mathrm{U}$ entre 0,2 y 6,2 confirma el origen magmático, de acuerdo con Rubatto (2017), porque solamente un fundido enriquecido en elementos raros e incompatibles permite alcanzar estas altas relaciones, lo cual es coherente con el origen asumido de la sienita nefelínica generada por un magmatismo intraplaca alcalino.

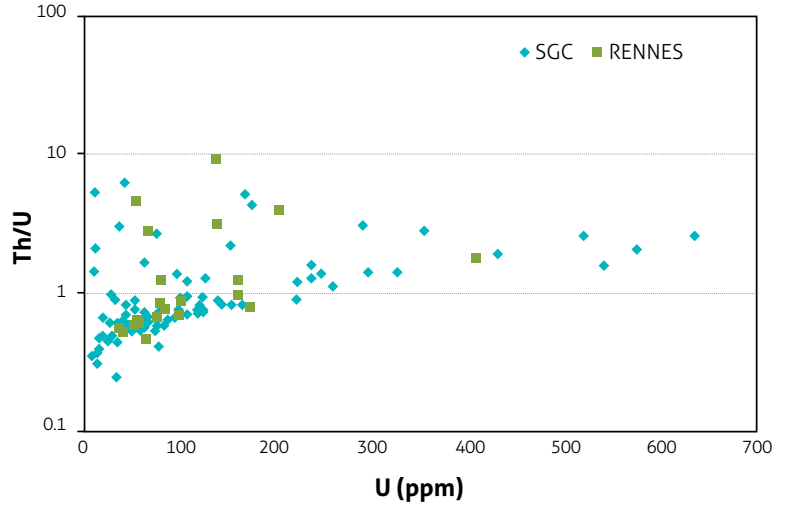

Figura 10. Relación Th/U versus contenido de $\mathrm{U}$ (ppm) de zircones magmáticos de la sienita. Fuente: autores

\section{Discusión de RESUltados}

\subsection{Edad de cristalización de la sienita nefelínica} Las edades ${ }^{206} \mathrm{~Pb} /{ }^{238} \mathrm{U}$ calculadas de un total de 92 análisis (Discordias < 5\%) en el LG-SGC por LA-ICP-MS, escogidas de circones de la sienita nefelínica de San José del Guaviare en la localidad del Jordán, muestran una distribución normal unimodal (figura 5) con una edad media de $608,5 \pm 1,0 \mathrm{Ma}( \pm 0,16 \%$, MSWD $=0,61)$.

Los datos discordantes (figura 7) se deben a una pérdida de $\mathrm{Pb}$, probablemente como consecuencia de la metamictización causada por el decaimiento de Th y U enriquecido en inclusiones minerales de hasta 10 micras. Un evento termal que afectó esta región durante el Ordovícico (Pinson, Hurley, Mencher y Fairbairn, 1962) puede haber facilitado la migración de uranio y plomo, y a la vez 
la recristalización en anillos discretos que se observa en los puntos con mayores edades discordantes.

Las edades concordia obtenidas en los laboratorios, LG - SGC con 92 análisis edad de 608,8 $\pm 1,3$ ( \pm 0,21\%) Ma y Laboratorio de Rennes con 24 análisis edad de 602 $\pm 3( \pm 0,5 \%)$ Ma con MSWD de 0,041 y 0,55$)$ y un promedio entre ambos laboratorios de 605,4 Ma sugieren la cristalización debajo de la temperatura de cierre de los circones durante el Ediacárico Temprano (Franco et al., 2018), considerablemente anteriores que las edades reportadas en la localidad de La Pizarra, con edades U/Pb en circón de 577,8 \pm 6,3 Ma (Ediacárico Tardío) para la cristalización y edades ${ }^{40} \mathrm{Ar} /{ }^{39} \mathrm{Ar}(\mathrm{HS})$ en biotita de $494 \pm 5$ $\mathrm{Ma}$ (Furoginiano) del enfriamiento debajo del punto de cierre de las biotitas (Arango et al., 2011 y 2012).

\subsection{Precisión analítica de los resultados LA-ICP- MS correspondientes a la sienita nefelínica de San José del Guaviare}

Los resultados de la datación de la sienita nefelínica usando LA-ICP-MS en los laboratorios del Servicio Geológico Colombiano y la de Universidad de Rennes, en Francia, se muestran en las figura 6 y 9 , con variaciones de 608,8 Ma a 602,0 Ma, respectivamente, con errores de 1,3 Ma y 3,0 Ma. La diferencia entre las edades calculadas para los dos laboratorios fue del 1,12\%.

Los materiales de referencia utilizados para evaluar tanto la exactitud como la precisión de la medición fueron Plesovice en la Universidad de Rennes, cuya edad de concordia obtenida fue de $336,5 \pm 5,5 \mathrm{Ma}( \pm 1,63 \%)$ y una diferencia con la edad esperada del $-0,19 \%$ (figura 9), y en el Servicio Geológico Colombiano, las edades obtenidas en el SGC (figura 8) fueron, con FC-5 Duluth, $1.103,4 \pm 3,9 \mathrm{Ma}(1.099 \pm 0,6 \mathrm{Ma}$, según Paces y Miller, 1993), y con Mount Dromedary, 100,10 $\pm 0,61 \mathrm{Ma}(99,12$ \pm 0,14 Ma, según Renne et al., 1998), con diferencias en las edades esperadas de $0,4 \%$ y $0,1 \%$ respectivamente. Las diferencias de los resultados obtenidos en los materiales de referencia evaluados por ambas organizaciones, tanto en error como en exactitud respecto a la edad esperada, están por debajo de las diferencias reveladas en estudios interlaboratorios, como los de Li, Liu, Su, Sun, Huang y Yi (2015) y Klötzli, Klötzli, Günes y Koslar (2009).

Los valores $2 \sigma$ de las mediciones concordantes con distribución normal indican una buena reproducibilidad o precisión interna. La diferencia de $6,8 \mathrm{Ma}(1,1 \%)$ del valor medio de las edades determinadas en ambos laboratorios serán objeto de estudios futuros. Las 92 mediciones escogidas en el SGC, de las cuales se intentó reproducir 24 mediciones en puntos cercanos de circones en Rennes, son finalmente una base estadística por debajo de las mínimo 100 series de medición que se espera en geocronología para tener un $2 \sigma \mathrm{m}$ robusto, $\sigma \mathrm{m}=\sigma / \sqrt{ } \mathrm{n}$, lo que podría sugerir la necesidad de aumentar la cantidad de las mediciones, para así seguir refinando la metodología y los protocolos.

Los resultados muestran en ambos laboratorios MSWD (mean square weighted deviation) menores que 1 (figuras 6 y 9). Considerando que MSWD $>2,5$ indican desequilibrio isotópico, este indicador de la bondad de ajuste derivado de la estadística chi-cuadrada reducida confirma una buena calidad de la reproducibilidad interna y externa de prueba $\chi^{2}$ de los valores medidos, indicando que los resultados de las mediciones representan datos geocronológicos coherentes y confirman que había equilibrio isotópico en el momento de cierre, y que el sistema quedó cerrado posteriormente. Los valores muy bajos indican, por su parte, incertidumbres sobreestimadas o que no reflejan la variación real de los datos, lo cual posiblemente sea consecuencia del esquema de reducción que se haya utilizado en cada laboratorio, como mencionan Spencer, Kirkland y Taylor (2016), es decir, baja dispersión de los resultados o incertidumbres sobreestimadas.

\section{Conclusiones}

Este estudio de datación U-Pb con LA-ICPMS de circones procedentes de la sienita nefelínica de la localidad del Jordán, Guaviare, Colombia, muestra que las edades obtenidas por los dos laboratorios son precisas, con incertidumbres o porcentajes de error bajos y diferencias entre edades de aproximadamente del $1 \%$. Lo mismo puede decirse de los materiales de referencia, estándares internacionales e internos.

Los megacristales de circón de la sienita nefelínica de San José del Guaviare tienen potencial como material interno de referencia, con edades ediacáricas de cristalización de entre $602 \pm 3$ Ma y 608,8 $\pm 1,3 \mathrm{Ma}$. Sin embargo, eso requiere una cuidadosa selección del material, 
teniendo en cuenta la presencia de zonación y anillos de recristalización de áreas con daño metamíctico en algunos cristales. Solamente los cristales de una fracción menor a $1 \mathrm{~cm}$ son aptos como materiales de referencia, porque presentan menos fracturas y daño metamíctico, para lo cual, una revisión mediante imágenes de catodoluminiscencia es indispensable.

Consideramos que tal selección vale la pena, debido al gran tamaño $(>0,5 \mathrm{~cm})$ y abundancia de los cristales, pues un montaje de la calidad adecuada puede ser utilizado durante varias rutinas de ablación (spot $30 \mu \mathrm{m}$ ) sin ser reemplazado, ya sea para sintonización del sistema LAICPMS o como material de referencia interno para control de calidad del laboratorio, y el cual posteriormente puede ser pulido para ser reutilizado.

Proyectamos la realización de análisis adicionales en otros laboratorios de manera sistemática y en colaboración con otros expertos para la obtención de resultados y la distribución de este mineral a otros laboratorios, incluyendo comparación mediante técnicas como TIMS, MC-SIMS y SHRIMP, eventualmente después de reparación térmica de los daños cristalinos durante $48 \mathrm{~h}$ con $\mathrm{T}$ de $800-1000{ }^{\circ} \mathrm{C}$ y abrasión mecánica o química (Mattinson, 2005) para aumentar el número de edades concordantes. Además de continuar disminuyendo los errores analíticos, es necesario indagar sobre las razones de la diferencia de edades de casi $1 \%$ entre los dos laboratorios, los efectos de la metamictización y posibles influencias de la meteorización y del ambiente oxidante sobre los cristales recolectados que facilitan migraciones y pérdidas de $\mathrm{U}$ y $\mathrm{Pb}$ y puedan influir así la zonación visible en catodoluminiscencia, la distribución espacial de otros elementos traza, con el objeto de verificar con más datos la aptitud de una fracción considerable de estos circones, abundantes como material de referencia. Los contenidos relativamente altos de Th, y por eso de ${ }^{208} \mathrm{~Pb}$, sugieren que también debe indagarse sobre el uso de este tercer grupo isotópico en la caracterización de los circones, y a la vez entender mejor el desarrollo geológico de esta parte del cratón amazónico y de sus sienitas nefelínicas, intrusivos subsiliciosos hasta ahora no reportados en otras partes del país.

De esta manera, el LG-SGC no solamente va acumulando experiencia en el cumplimiento de los protocolos internacionales, el refinamiento de la metodología y su ampliación a otros sistemas isotópicos, sino que puede usar cada vez más el creciente parque analítico y la capa de investigadores existentes en el país para hacer aportes geocientíficos relevantes sobre la materia tanto en Colombia como a escala mundial.

\section{REFERENCIAS BIBLIOGRÁFICAS}

Arango, M. I., Zapata García, G. y Martens, U. (2012). Caracterización petrográfica, geoquímica y edad de la sienita nefelínica de San José del Guaviare. Boletín de Geología, 34, 15-26.

Arango, M., Nivia, A., Zapata, G., Giraldo, M., Bermúdez, J. y Albarracín, H. (2011). Geología y geoquímica de la plancha 350, San José del Guaviare. Memoria. Medellín: Servicio Geológico Colombiano.

Campbell, I. H., Reiners, P. W., Allen, C. M., Nicolescu, S. y Upadhyay, R. (2005). He-Pb double dating of detrital zircons from the Ganges and Indus Rivers: Implication for quantifying sediment recycling and provenance studies. Earth and Planetary Science Letters, 237 (3-4), 402-432. Doi: https://doi.org/10.1016/j. epsl.2005.06.043.

Campos Rodríguez, H. R. (2017). Caracterización geoquímica y mineralógica de la sienita nefelínica de San José del Guaviare y minerales asociados con elementos raros (ER). Trabajo de Grado. Bogotá: Universidad Nacional de Colombia.

Chang, Z., Vervoort, J. D., McClelland, W. C. y Knaack, C. (2006). U-Pb dating of zircon by LA-ICP-MS. Geochemistry, Geophysics, Geosystems, 7 (5). Doi: https://doi.org/10.1029/2005GC001100.

Corfu, F., Hanchar, J. M., Hoskin, P. W. O. y Kinny, P. (2003). Atlas of zircon textures. Reviews in Mineralogy and Geochemistry, 53 (1): 469-500. Doi: https://doi. org/10.2113/0530469.

Crowley, J. L., Schoene, B. y Bowring, S. A. (2007). U$\mathrm{Pb}$ dating of zircon in the Bishop Tuff at the millennial scale. Geology 35 (12), 1123-1126. Doi: https://doi.org/10.1130/G24017A.1.

Fedo, C. M., Sircombe, K. N. y Rainbird, R. H. (2003). Detrital zircon analysis of the sedimentary record. Reviews in Mineralogy and Geochemistry, 53 (1), 1-25. Doi: https://doi.org/10.2113/0530277. 
Finch R. J. y Hanchar J. M. (2005). Structure and Chemistry of Zircon and Zircon-Group Minerals. Reviews in Mineralogy and Geochemistry 53 (1): 1-25. Doi: https://doi.org/10.2113/0530001.

Franco, J. A., Muñoz, J. A., Piraquive, A., Bonilla, A., Cramer, T. y Campos, H. (2018). Geochronology of the nepheline syenite of el Jordán, Guaviare, Colombia, evidences of Neoproterozoic-Cambrian intraplate magmatism and its implications during Pan-African tectonics in Western Gondwana. En EGU2018 general assembly, Vienna, EGU2018, 20. Disponible en https://meetingorganizer.copernicus.org/EGU2018/ EGU2018-10861.pdf.

García Romero, D. F. (2015). Petrografía y geoquímica de las rocas aflorantes al sur de San José del Guaviare. Trabajo de Grado. Bogotá: Universidad Nacional de Colombia.

Gehrels, G. E., Valencia, V. A. y Ruiz, J. (2008). Enhanced precision, accuracy, efficiency, and spatial resolution of $\mathrm{U}-\mathrm{Pb}$ ages by laser ablation-multicollector-inductively coupled plasma-mass spectrometry. Geochemistry, Geophysics, Geosystems, 9 (3). Doi: https://doi.org/10.1029/2007GC001805.

Hanchar, J. M. y Miller, C. F. (1993). Zircon zonation patterns as revealed by cathodoluminescence and backscattered electron images: Implications for interpretation of complex crustal histories. Chemical Geology, 110 (1-3), 1-13. Doi: https://doi.org/10.1016/0009-2541(93)90244-D.

Harrison, T. M., Watson, E. B. y Aikman, A. B. (2007). Temperature spectra of zircon crystallization in plutonic rocks. Geology, 35 (7), 635-638. Doi: https://doi.org/10.1130/G23505A.1

Jackson, S. E., Pearson, N. J., Griffin, W. L. y Belousova, E. A. (2004). The application of laser ablation-inductively coupled plasma-mass spectrometry to in situ $\mathrm{U}-\mathrm{Pb}$ zircon geochronology. Chemical Geology, 211 (1-2), 47-69. Doi: https://doi.org/10.1016/j.chemgeo.2004.06.017.

Jaffey, A. H., Flynn, K. F., Glendenin, L. E., Bentley, W. C. y Essling, A. M. (1971). Precision measurement of half-lives and specific activities of ${ }^{235} \mathrm{U}$ and ${ }^{238} \mathrm{U}$. Physical Review Section C, 4 (5). Doi: https://doi. org/10.1103/PhysRevC.4.1889.
Klötzli, U., Klötzli, E., Günes, Z. y Koslar, J. (2009). Accuracy of laser ablation $\mathrm{U}-\mathrm{Pb}$ zircon dating: Results from a test using five different reference zircons. Geostandards and Geoanalytical Research, 33 (1), 5-15. Doi: https://doi.org/10.1111/j.1751-908X.2009.00921.x.

Koschek, G. (1993). Origin and significance of the SEM cathodoluminescence from zircon. Journal of Microscopy, 171 (3), 223-232. Doi: https://doi. org/10.1111/j.1365-2818.1993.tb03379.x.

Košler, J. y Sylvester, P. J. (2003). Present trends and the future of zircon in geochronology: laser ablation ICPMS. Reviews in Mineralogy and Geochemistry, 53, 243-275. Doi: https://doi.org/10.2113/0530243.

Li, X., Liu, X., Liu, Y., Su, L., Sun, W., Huang, H. y Yi, K. (2015). Accuracy of LA-ICPMS zircon U-Pb age determination: An inter-laboratory comparison. Science China Earth Sciences, 58 (10), 1722-1730. Doi: https:// doi.org/10.1007/s11430-015-5110-x.

Ludwig, K. R. (2012). User's manual for Isoplot 3.75, a geochronological toolkit for Microsoft Excel. Berkeley Geochronology Center Special Publication, v. 5, 1-72.

Mattinson, J. M. (2005). Zircon U-Pb chemical abrasion ("CA-TIMS") method: Combined annealing and multi-step partial dissolution analysis for improved precision and accuracy of zircon ages. Chemical Geology, 220 (1-2), 47-66. Doi: https://doi.org/10.1016/j. chemgeo.2005.03.011.

Paces, J. B. y Miller, J. D. (1993). Precise U-Pb ages of Duluth Complex and related mafic intrusions, northeastern Minnesota: Geochronological insights to physical, petrogenetic, paleomagnetic, and tectonomagmatic processes associated with the $1.1 \mathrm{Ga}$ Midcontinent Rift System. Journal of Geophysical Research Solid Earth, 98 (B8), 13997-14013. Doi: https:// doi.org/10.1029/93JB01159.

Paton, C., Hellstrom, J., Paul, B., Woodhead, J. y Hergt, J. (2011). Iolite: Freeware for the visualisation and processing of mass spectrometric data. Journal of Analytical Atomic Spectrometry, 12, 2508-2518. Doi: https:// doi.org/10.1039/C1JA10172B.

Peña Urueña, M. L., Muñoz Rocha, J. A. y Urueña, C. L. (2018). Laboratorio de Geocronología en el Servicio Geológico Colombiano: avances sobre datación $\mathrm{U}-\mathrm{Pb}$ en circones mediante la técnica LA-ICP- 
MS. Boletín Geológico, 44, 39-56. Doi: https://doi. org/10.32685/0120-1425/boletingeo.44.2018.7.

Petrus, J. A. y Kamber, B. S. (2012). Vizual age: A novel approach to laser ablation ICP-MS U-Pb geochronology data reduction. Geostandards and Geoanalytical Research, 36 (3), 247-270. Doi: https://doi. org/10.1111/j.1751-908X.2012.00158.x.

Pinson W. H, J. R., Hurley, P. M., Mencher, E. y Fairbairn, H. W. (1962). K-Ar AND Rb-Sr Ages of Biotites from Colombia, South America. GSA Bulletin, 73(7), 907-910. Doi: https://doi. org/10.1130/0016-7606(1962)73[907:KARAOB]2.0. $\mathrm{CO} ; 2$

Renne, P. R., Swisher, C. C., Deino, A. L., Karner, D. B., Owens, T. L. y De Paolo, D. J. (1998). Intercalibration of standards, absolute ages and uncertainties in 40Ar/39Ar dating. Chemical Geology, 145 (1-2), 117-152. Doi: https://doi.org/10.1016/S00092541(97)00159-9.

Rubatto, D. (2002). Zircon trace element geochemistry: Partitioning with garnet and the link between $\mathrm{U}-\mathrm{Pb}$ ages and metamorphism. Chemical Geology, 184 (1-2), 123-138. Doi: https://doi.org/10.1016/S00092541(01)00355-2.

Rubatto, D. (2017). Zircon: The metamorphic mineral. Reviews in Mineralogy and Geochemistry, 83 (1), 261295. Doi: https://doi.org/10.2138/rmg.2017.83.9.

Rutherford, E. y Soddy, F. (1903). Radioactive change. The London, Edinburgh, and Dublin Philosophical Magazine and Journal of Science, 5 (29), 576-591. Doi: https://doi.org/10.1080/14786440309462960.

Schaltegger, U., Fanning, C. M., Günther, D., Maurin, J. C., Schulmann, K. y Gebauer, D. (1999). Growth, annealing and recrystallization of zircon and preservation of monazite in high-grade metamorphism: conventional and in-situ U-Pb isotope, cathodoluminescence and microchemical evidence. Contributions to Mineralogy and Petrology, 134 (2-3), 186-201. Doi: https://doi.org/10.1007/s004100050478.

Schmitz, M. D. y Schoene, B. (2007). Derivation of isotope ratios, errors and error correlations for $\mathrm{U}-\mathrm{Pb}$ geochronology using ${ }^{205} \mathrm{~Pb}-{ }^{235} \mathrm{U}-\left({ }^{233} \mathrm{U}\right)$-spiked isotope dilution thermal ionization mass spectrometric data. Geochemistry, Geophysics and Geosystems, 8 (8). Doi: https://doi.org/10.1029/2006GC001492.
Schoene, B. (2014). U-Th-Pb Geochronology. Vol. 4. Princeton: Princeton University Press. Doi: https:// doi.org/10.1016/B978-0-08-095975-7.00310-7.

Shannon, R. D. (1976). Revised effective ionic radii in halides and chalcogenides. Acta Crystallographica Section A, A32, 751-767. Doi: https://doi.org/10.1107/ S0567739476001551.

Sláma, J., Košler, J., Condon, D. J., Crowley, J. L., Gerdes, A., Hanchar, J. M. et al. (2008). Plešovice zircon: A new natural reference material for $\mathrm{U}-\mathrm{Pb}$ and $\mathrm{Hf}$ isotopic microanalysis. Chemical Geology, 249 (1-2), 1-35. Doi: https://doi.org/10.1016/j.chemgeo.2007.11.005.

Solari, L., Gómez Tuena, A., Bernal, J., Pérez Arvizu, O. y Tanner, M. (2010). U-Pb zircon geochronology with an integrated LA-ICPMS microanalitycal workstation: Achievements in precision and accuracy. Geostandards and Geoanalytical Reseach, 34 (1), 5-18. Doi: https://doi.org/10.1111/j.1751-908X.2009.00027.x.

Spencer, C. J., Kirkland, C. L. y Taylor, R. J. M. (2016). Strategies towards statistically robust interpretations of in situ U-Pb zircon geochronology. Geoscience Frontiers, 7 (4), 581-589. Doi: https://doi. org/10.1016/j.gsf.2015.11.006.

Sylvester, P. J. (2001). Data reduction software for LAICP-MS: Appendix. En Laser Ablation-ICP-mass spectrometry in the Earth sciences: Principles and applications (short course series). Ottawa: Mineralogical Association of Canada.

Tera, F. y Wasserburg, G. J. (1972). U-Th-Pb systematics in lunar highland samples from the Luna 20 and Apollo 16 missions. Earth and Planetary Science Letters, 17 (1), 36-51. Doi: https://doi.org/10.1016/0012821X(72)90257-9.

Tera, F. y Wasserburg, G. J. (1975). Precise isotopic analysis of lead in picomole and subpicomole quantities. Analytical Chemistry, 47 (13), 2214-2220. Doi: https:// doi.org/10.1021/ac60363a036.

Trumpy, D. (1943). Pre-cretaceous of Colombia. Bulletin of the Geological Society of America, 54 (9), 1281-1304. Doi: https://doi.org/10.1130/GSAB-54-1281.

Turekian, K. K. y Wedepohl, K. H. (1961). Distribution of the elements in some major units of the earth's crust. GSA Bulletin, 72 (2), 175-192. Doi: https://doi.org/10 .1130/0016-7606(1961)72[175:DOTEIS]2.0.CO;2. 
Watson, E. B. y Harrison, T. M. (2005). Zircon thermometer reveals minimum melting conditions on earliest Earth. Science, 308 (5723), 841-844. Doi: https://doi.org/10.1126/science.1110873.

Watson, E. B., Wark, D. A. y Thomas, J. B. (2006). Crystallization thermometers for zircon and rutile. Contributions to Mineralogy and Petrology, 151, 413-433. Doi: https://doi.org/10.1007/s00410-006-0068-5.

Wedepohl, K. H. (1995). The composition of the continental crust. Geochimica et Cosmochimica Acta, 59
(7), 1217-1232. Doi: https://doi.org/10.1016/00167037(95)00038-2.

Wetherill, G. W. (1956). Discordant uranium-lead ages, I. Transactions, American Geophysical Union, 37 (3), 320326. Doi: https://doi.org/10.1029/TR037i003p00320.

Yang, B., Luff, B. J. y Townsend, P. D. (1992). Cathodoluminescence of natural zircons. Journal of Physics: Condensed Matter, 4 (25), 5617. Disponible en http://stacks.iop.org/0953-8984/4/i=25/a=015. 